\title{
Espectros Combinados en Subálgebras de Álgebras Topológicas
}

Tesis Doctoral Presentada por Armando Velázquez González

Asesor de Tesis

Dr. Antoni Wawrzyńczyk 


\section{"Espectros combinados en subálgebras de álgebras topológicas"}

Tesis que presenta:

Armando Velázquez González

Para obtener el grado de

\section{Doctor en Ciencias (Matemáticas).}

Asesor: Dr. Antoni Wawrzyńczyk.

Enero del 2005.

Univeridad Autónoma Metropolitana- Iztapalapa.

División de Ciencias Básicas e Ingeniería. 


\section{AGRADECIMIENTOS}

Por medio de las siguientes líneas quiero expresar mi admiración e infinito amor a mi madre, ya que siendo una familia numerosa y humilde ha logrado, a mis hermanos y a mi, guiarnos por la senda adecuada a pesar de las adversidades.

Agradezco a todos mis hermanos su incondicional apoyo, sin el mi objetivo no se hubiese realizado.

Quiero expresar mi agradecimiento a mi tío Fidel Hernandez (q.e.p.d.), por que gracias a sus consejos y a su incondicional apoyo me guió por buen camino.

En la misma medida agradezco al Sr. José María Montoya (q.e.p.d.), quien en los momentos más difíciles fue un gran soporte para mi familia.

A todos mis sobrinos y muy en especial a Osvaldo Loza Velázquez y su familia, por escucharme y compartir mis momentos de preocupación y de alegría.

A mis tios Esperanza González García (q.e.p.d.) y Adán González Salas (q.e.p.d.), por apoyarme en mi proyecto académico.

A la Dra. Lourdes Palacios Fábila por todo el apoyo que me brindó a lo largo de mis estudios doctorales y por la gran calidad humana que posee.

A mis amigos Dr. Baltazar Aguirre, M. en C. Oswaldo González Gaxiola, Dr. Joaquín Tey, M. en C. Isaí Moreno Roque, por hacer más placentera mi estancia doctoral y por brindarme su amistad.

A los Doctores Juan Hector Arredondo, Roberto Quezada, Richard Wilson, María José Arrollo, por ser exelentes profesores y muy buenas personas.

Al Consejo Nacional de Ciencia y Tecnología por el apoyo económico recibido para cumplir este proyecto.

Al Jefe del departamento Dr. Carlos Signoret Poillon, por todo el apoyo recibido y por su gran calidad humana.

A la Dra. Shirley Bromberg, por todo el apoyo recibido a lo largo de mis estudios doctorales.

Al Dr. Antoni Wawrzyńczyk, por dirigir este trabajo, por soportarme, por ser un excelente profesor y sobre todo por ser un excelente ser humano.

Gracias a la Universidad Autónoma Metropolitana-Iztapalapa, al Departamento de Matemáticas de la UAM-I, por acogerme en su seno. Espero responder con creces todo el apoyo recibido. 


\section{Índice General}

INTRODUCCIÓN 2

1 Álgebras de Banach 4

1.1 Conceptos básicos de álgebras de Banach . . . . . . . . . . . . . 4

1.2 Conceptos básicos de álgebras de Banach conmutativas . . . . . . 11

2 La propiedad de la proyección de una familia de ideales en $\begin{array}{ll}\text { subálgebras de álgebras de Banach } & 18\end{array}$

2.1 Espectro combinado definido por una familia de ideales . . . . . 19

2.2 La propiedad de la proyección de $I_{E}^{l}(A) \ldots \ldots$. . . . . . . . 20

2.3 Subespectro asociado a $I_{E}^{l}(A) \ldots \ldots$. . . . . . . . . . 22

2.4 Caso $A$ conmutativa . . . . . . . . . . . . . . . . . . 24

2.5 Un teorema de separación . . . . . . . . . . . . . . . 27

3 Conceptos básicos de álgebras topológicas 29

3.1 Conceptos básicos . . . . . . . . . . . . . . . . 29

3.2 Ejemplos de álgebras topológicas . . . . . . . . . . . . . . . . 33

3.3 Topologías definidas por semi-normas submultiplicativas . . . . . 36

3.4 Ciertas clases particulares de álgebras topológicas . . . . . . . . . 40

4 Fórmula del mapeo espectral para álgebras de Waelbroeck y sus subálgebras $\quad 49$

4.1 Los ideales de $A \subset E$ que consisten de elementos no invertibles en $E$. . . . . . . . . . . . . . . . . . . . . . . . . . . . . . . . 50

4.2 Casos especiales y aplicaciones . . . . . . . . . . . . 51

5 La propiedad de la proyección de una familia de ideales y la fórmula del mapeo espectral en álgebras localmente m-convexas 53

$\begin{array}{ll}\text { Bibliografía } & 56\end{array}$ 


\section{INTRODUCCIÓN}

Un número complejo $\lambda$ pertenece al espectro de un operador $T$ si $T-\lambda I$ no es invertible. Esta definición se aplica igualmente al caso de un operador en espacios de dimensión finita, normados, de Hilbert o de Banach. Mientras que en dimensión finita cada elemento del espectro corresponde a un vector propio del operador $T$, en dimensión infinita la no invertibilidad de un operador puede tener causas diversas. Estos fenómenos crean la necesidad de considerar en el espectro puntos "más malos" y puntos "menos malos" o, en otras palabras varios tipos de espectros.

La teoría espectral de álgebras de Banach, donde se observan los mismos fenómenos, alcanzó un nivel tan desarrollado en la década de los setenta que resultó necesario darle forma axiomática.

En el caso clásico del álgebra conmutativa $C(K)$ de funciones continuas sobre un conjunto compacto $K$, el espectro de una función $f \in C(K)$ coincide con el rango de la función.

Dada una $k$ - upla de funciones $f_{1}, \ldots, f_{k} \in C(K)$, el rango de la aplicación $K \rightarrow \mathbb{C}^{k}, x \rightarrow\left(f_{1}(x), \ldots, f_{k}(x)\right)$, es un subconjunto compacto de $\mathbb{C}^{k}$, el cual es el ejemplo básico de "espectro combinado" o espectro multivariable en un álgebra de Banach.

En el caso de un álgebra de Banach $E$, no necesariamente conmutativa, el espectro combinado fundamental de una $k$-upla de elementos $b_{1}, \ldots, b_{k}$, que conmutan entre sí, fue definida por R. Harte en 1973 de la siguiente manera: $\left(\lambda_{1}, \ldots, \lambda_{k}\right) \in S p\left(b_{1}, \ldots, b_{k}\right)$ si por lo menos uno de los ideales, izquierdo o derecho, generado por $b_{1}-\lambda_{1}, \ldots, b_{k}-\lambda_{k}$ es propio. En el caso de un solo elemento, el espectro de Harte coincide con el espectro usual.

Por las mismas razones que se han dado en el caso del espectro de un solo elemento, también en el caso del espectro combinado existe la necesidad de introducir varios tipos de espectro tales como: espectro puntual aproximativo, espectro esencial, espectro de defecto, espectro de Taylor, por mencionar algunos.

Según la axiomática de W. Żelazko [Ż7], un espectro combinado o un subespectro es una aplicación que asocia a una $k-u p l a$ de elementos $a_{1}, \ldots a_{k} \in E$, que conmutan mutuamente, un conjunto compacto $\widetilde{\sigma}\left(a_{1}, \ldots a_{k}\right) \in \mathbb{C}^{k}$ de tal manera que:

i) $\tilde{\sigma}\left(a_{1}, \ldots a_{k}\right) \subset \prod_{i=1}^{k} \sigma\left(a_{i}\right)$, donde $\sigma\left(a_{i}\right)$ es el espectro usual de $a_{i}$ en $E$;

ii) $P\left(\widetilde{\sigma}\left(a_{1}, \ldots a_{k}\right)\right)=\widetilde{\sigma}\left(P\left(a_{1}, \ldots a_{k}\right)\right)$, para cualquier mapeo polinomial $P: \mathbb{C}^{k} \rightarrow \mathbb{C}^{m}$.

Varios de los espectros antes mencionados se obtienen de acuerdo al siguiente esquema: en el álgebra $E$ distinguimos un conjunto de elementos "buenos" $R$ 
( elementos inveribles, en el caso de Harte). Luego definimos $\left(\lambda_{1}, \ldots, \lambda_{k}\right) \in$ $S p_{R}\left(a_{1}, \ldots, a_{k}\right)$ cuando el ideal derecho o izquierdo, generado por $\left(a_{1}-\lambda_{1}, \ldots\right.$, $\left.a_{k}-\lambda_{k}\right)$ en $E$, no se intersecta con $R$.

El resultado principal de la tesis presenta un método para obtener un conjunto $R$ que conduce a varios subespectros conocidos y otros espectros no estudiados hasta ahora. Este método consiste en considerar un álgebra $E$ que contiene a $A$ como subálgebra y tomar como $R$ el conjunto de elementos de $A$ no invertibles en $E$. Sorprendentemente este método genera una clase muy amplia de subespectros no solamente para álgebras de Banach sino también para álgebras topológicas de Waelbroeck.

En los capítulos 1 y 3 damos definiciones y resultados básicos para álgebras de Banach complejas con unidad, álgebras de Banach conmutativas complejas con unidad y álgebras topológicas complejas con unidad.

En el capítulo 2 obtenemos un hecho puramente algebraico acerca del espectro combinado, definido por una familia de ideales izquierdos (derechos) de un álgebra con unidad (Teorema 2.1.1). También demostramos que la familia $I_{E}^{l}(A)$ de ideales izquierdos de $A$ que consisten de elementos no invertibles en $E$, donde $E$ es un álgebra de Banach con unidad y $A$ es una subálgebra de $E$ con unidad, tiene la propiedad de la proyección. Según la axiomática introducida por Żelazko, se prueba que

$$
S p_{E}\left(a_{1}, \ldots, a_{k}\right)=\left\{\left(\lambda_{1}, \ldots, \lambda_{k}\right) \in \mathbb{C}^{k}: \exists I \in I_{E}^{l}(A), a_{i}-\lambda_{i} \in I, 1 \leq i \leq k\right\}
$$

es un subespectro.

En el capítulo 4 demostramos que la familia $I_{E}^{l}(A)$ también satisface la propiedad de la proyección. donde $E$ es una $Q$ - álgebra compleja con unidad y $A$ es una subálgebra de $E$ con unidad. Si $E$ es un álgebra de Waelbroeck localmente convexa compleja con unidad, entonces obtenemos que el espectro combinado $S p_{I_{E}^{l}(A)}\left(a_{1}, \ldots a_{k}\right)$ satisface la fórmula del mapeo espectral.

Finalmente, en el capítulo 5 consideramos una clase de espectros combinados sobre álgebras localmente $m$ - convexas complejas con unidad, definidos para una familia de ideales izquierdos o derechos apropiados sobre tales álgebras, demostrando la propiedad de la proyección y la fórmula del mapeo espectral. 


\section{Capítulo 1}

\section{Álgebras de Banach}

Este capítulo contiene definiciones y resultados básicos de la teoría de álgebras de Banach y álgebras de Banach conmutativas. Para más detalles ver [BD] o algunos otros libros sobre álgebras de Banach ( [Ri],[Z5],[Pa] ).

En todo este trabajo por álgebra siempre entenderemos un álgebra compleja con unidad $e$.

\subsection{Conceptos básicos de álgebras de Banach}

Definición 1.1.1. Un álgebra $E$ es un espacio vectorial complejo junto con una operación binaria, llamada multiplicación, $(x, y) \rightarrow x y$ de $E \times E \rightarrow E$ la cual satisface las siguientes condiciones (para todo $x, y, z \in E, \lambda \in \mathbb{C}$ ):

i) $x(y z)=(x y) z$;

ii) $x(y+z)=x y+x z,(x+y) z=x z+y z$;

iii) $(\lambda x) y=\lambda(x y)=x(\lambda y)$;

$i v)$ existe un elemento único $e \in E$ tal que ex $=x e=x$, para todo $x \in E$.

Es fácil probar que el elemento unidad está determinado en forma única. Para un número complejo $\lambda$, el símbolo $\lambda$ también denotará al elemento $\lambda e$ del álgebra.

Definición 1.1.2. Sea $E$ un álgebra. Una semi-norma submultiplicativa en $E$ es una función $\|\cdot\|: E \rightarrow(0, \infty), x \rightarrow\|x\|$ la cual satisface (para todo $x, y \in E, \lambda \in \mathbb{C})$ :

i) $\quad\|\lambda x\|=|\lambda|\|x\|$;

ii) $\|x+y\| \leq\|x\|+\|y\|$; 
iii) $\|x y\| \leq\|x\|\|y\|$;

iv) $\|e\|=1$.

Definición 1.1.3. Una norma submultiplicativa en $E$ es una semi-norma submultiplicativa en E tal que:

v) si $\|x\|=0$ entonces $x=0$.

Definición 1.1.4. Un álgebra normada es un par $(E,\|\cdot\|)$ donde $E$ es un álgebra y \|·\| es una norma submultiplicativa en E. Un álgebra de Banach es un álgebra normada que es completa en la topología definida por la norma (en otras palabras, $(E,\|\cdot\|)$, considerado como un espacio vectorial, es un espacio de Banach).

Ejemplos. Hay muchos ejemplos de álgebras de Banach que aparecen naturalmente en análisis funcional.

1) El ejemplo más importante de un álgebra de Banach es el álgebra $L(X)$ que consiste de todos los operadores lineales acotados que actúan sobre un espacio de Banach $X, \operatorname{dim} X \geq 1$, con las operaciones algebraicas naturalmente definidas y con la norma $\|T\|=\sup \{\|T x\|:\|x\|=1\}$. El elemento unidad en $L(X)$ es el operador identidad $I$ definido por $I x=x$, para todo $x \in X$.

En particular, si $\operatorname{dim} X=n$ entonces $L(X)$ puede ser identificado con el álgebra de las matrices complejas de $n \times n$.

2) Sean $K$ un espacio compacto y $C(K)$ el álgebra de todas las funciones continuas de valor complejo definidas sobre $K$. Entonces $C(K)$, con la norma supremo $\|f\|=\sup \{|f(x)|: x \in K\}$, es un álgebra de Banach.

3) Sea $E$ un conjunto no vacío. El conjunto de todas las funciones acotadas de valor complejo definidas en $E$, con las operaciones algebraicas definidas puntualmente y la norma supremo, es un álgebra de Banach.

Similarmente, sea $L^{\infty}$ el conjunto de todas las funciones medibles y acotadas de valor complejo, definidas en el conjunto de los números reales, con la medida de Lebesgue. Entonces $L^{\infty}$, junto con las operaciones algebraicas definidas puntualmente y la norma supremo, es un álgebra de Banach.

4) Sea $D$ el disco unitario abierto en el plano complejo. Denotemos por $H^{\infty}$ al álgebra de todas las funciones que son analíticas y acotadas en $D$. Sea $A(D)$ el álgebra de todas las funciones que son continuas en $\bar{D}$ y analíticas en $D$. Ambas álgebras $A(D)$ y $H^{\infty}$, con la norma supremo, son ejemplos importantes de álgebras de Banach.

Observación. Frecuentemente la condición (iii) de la definición 1.1.2 es reemplazada por una condición más débil de continuidad de la multiplicación. Sin embargo, es posible reducir estas definiciones más generales de álgebras de Banach a la definición 1.1.2. 
Definición 1.1.5. Sean $A$ y $B$ dos álgebras. Un mapeo lineal $h: A \rightarrow B$ es llamado un homomorfismo si $h(x y)=h(x) h(y)$ y $h\left(e_{A}\right)=e_{B}$.

Un subconjunto $A$ de un álgebra $E$ es llamada una subálgebra si es cerrado bajo las operaciones algebraicas (es decir, $A$ es un subespacio vectorial de $E$, y si $x, y \in A$ entonces $x y \in A$ ).

Si $A$ es una subálgebra cerrada de un álgebra de Banach $E$, entonces $A$, con la norma restringida, es un álgebra de Banach.

Definición 1.1.6. Sean $E_{1}$ y $E_{2}$ dos álgebras de Banach. Un homomorfismo $h: E_{1} \rightarrow E_{2}$ es llamado un homomorfismo topológico si $h$ es continuo. Un isomorfismo $h: E_{1} \rightarrow E_{2}$ es llamado un isomorfismo topológico siempre que $h$ sea un homeomorfismo entre $E_{1}$ y $h\left(E_{1}\right)$. Un homomorfismo $h: E_{1} \rightarrow E_{2}$ es llamado isométrico si $\|h(x)\|=x$, para toda $x \in E_{1}$.

Para un álgebra normada $A$ existe su completación, un álgebra de Banach $E$ determinada en forma única (salvo un isomorfismo isométrico), tal que $A$ es una subálgebra densa de $E$.

A partir de este momento, un homomorfismo (isomorfismo) entre álgebras de Banach significará un homomorfismo (isomorfismo) topológico. Si la continuidad no es asumida, hablaremos de homomorfismo (isomorfismo) algebraico.

Definición 1.1.7. Sea $E$ un álgebra. Un conjunto $I \subset E$ es llamado un ideal izquierdo (derecho) en $E$ si $I$ es un subespacio de $E$ y ax $\in I(x a \in I)$, para todo $x \in E, a \in I$. El conjunto $I$ es un ideal bilateral en $E$ si $I$ es tanto un ideal izquierdo como un ideal derecho en E. Un ideal (izquierdo, derecho o bilateral) $I \subset E$ es llamado propio si $I$ es diferente de $E$.

Sea $I$ un ideal bilateral propio de un álgebra $E$. Los subconjuntos de $E$ de la forma $x+I$, con $x \in E$, son llamados clases laterales de $I$ en $E$. Dos clases laterales distintas siempre son ajenas; su suma también es una clase lateral. Dado que $I$ es un ideal bilateral se sigue que el producto $(x+I)(y+I)$, de dos clases laterales, siempre está contenido en alguna clase lateral, la cual será llamada el producto de las clases laterales $x+I$ e $y+I$. Finalmente, un múltiplo escalar de una clase lateral es nuevamente una clase lateral. Así, el conjunto de todas las clases laterales de $I$ en $E$ tiene la estructura de un álgebra. Esta álgebra será denotada por $E / I$ y será llamada el álgebra cociente de $E$ módulo $I$. El mapeo $h: E \rightarrow E / I$, dado por $x \rightarrow x+I$, será llamado el homomorfismo natural de $E$ sobre $E / I$.

Un ideal bilateral propio $I$ de un álgebra $E$ es llamado maximal si no está contenido propiamente en algún otro ideal propio. Todo ideal propio en un álgebra conmutativa está contenido en algún ideal maximal; lo mismo se cumple para álgebras no conmutativas, con la observación de que los ideales izquerdo y derecho se deben tratar por separado (el correspondiente ideal maximal es también izquierdo o derecho, según sea el caso). La prueba de estos hechos es 
una consecuencia inmediata del Lema de Zorn-Kuratowsky. En adelante, por un ideal propio entenderemos un ideal bilateral propio.

Lema 1.1.8. Sean $E_{1}, E_{2}$ dos álgebras de Banach y $h: E_{1} \rightarrow E_{2}$ un homomorfismo. Entonces ker $h=\left\{x \in E_{1}: h(x)=0\right\}$ es un ideal cerrado en $E_{1}$.

Demostración. Es claro que ker $h$ es un ideal. Dado que $\{0\}$ es cerrado en $\mathbb{C}$ y ker $h=h^{-1}(\{0\}), h$ es continua, entonces ker $h$ es cerrado en $E_{1}$.

Definición 1.1.9. Sean $E$ un álgebra y $x, z \in E$. $z$ es llamado un inverso izquierdo (derecho) de $x$ si $z x=e(x z=e)$. Si $z$ es tanto inverso izquierdo como inverso derecho de $x$, entonces $z$ es llamado un inverso de $x$.

Si $x$ tiene un inverso $z$, éste es único. En efecto, si $\widetilde{z}$ es otro inverso de $x$ entonces $\widetilde{z}=\widetilde{z} e=\widetilde{z}(x z)=(\widetilde{z} x) z=e z=z$.

Sea $E$ un álgebra. Un elemento de $E$ para el cual existe un inverso (derecho, izquierdo), será llamado invertible (derecho, izquierdo). El inverso de un elemento invertible $x$ será denotado por $x^{-1}$. El conjunto de todos los elementos invertibles en $E$ será denotado por $G(E)$. Similarmente, el conjunto de todos los elementos invertibles izquierdos (invertibles derechos) en $E$ serán denotados por $G^{l}(E)\left(G^{r}(E)\right)$; es decir

$$
\begin{aligned}
G^{l}(E) & =\{x \in E: \text { existe } y \in E \text { tal que } y x=e\} \\
G^{r}(E) & =\{x \in E: \text { existe } y \in E \text { tal que } x y=e\} .
\end{aligned}
$$

Claramente $G(E)=G^{l}(E) \cap G^{r}(E)$.

Es fácil ver que un elemento $x \in E$ tiene un inverso izquierdo (derecho) si, y sólo si, $x$ no está contenido en un ideal izquierdo (derecho) propio.

Teorema 1.1.10. Sea E un álgebra de Banach.

i) Si $x \in E,\|x\|<1$, entonces $e-x \in G(E)$;

ii) Los conjuntos $G(E), G^{l}(E)$ y $G^{r}(E)$ son abiertos;

iii) El mapeo $x \rightarrow x^{-1}$ es continuo en $G(E)$.

Demostración. (i) Si $\|x\|<1$ entonces $\left\|x^{n}\right\| \leq\|x\|^{n}<1$. Así, la serie $\sum_{n=0}^{\infty} x^{n}$ es convergente en $E$ y

$$
\begin{aligned}
(e-x) \sum_{n=0}^{\infty} x^{n} & =\sum_{n=0}^{\infty} x^{n}(e-x)=\lim _{n \rightarrow \infty}\left\{\sum_{j=0}^{n} x^{j}-\sum_{j=0}^{n} x^{j+1}\right\} \\
& =\lim _{n \rightarrow \infty}\left(e-x^{n+1}\right)=e .
\end{aligned}
$$


ii) Sea $x \in G^{l}(E)$, demostremos que existe $U$ vecindad del cero tal que $x+U \subset G^{l}(E)$. Sean $y \in E$, tal que $y x=e$, y $U=\left\{u \in E:\|u\|<\|y\|^{-1}\right\}$. Sea $u \in U$, como $y x=e$ entonces $y(x+u)=e+y u$. Pero $\|y u\| \leq\|y\|\|u\|<1$. En consecuencia, por el inciso $(i), y(x+u)$ es invertible y $(y(x+u))^{-1} y(x+u)=e$. Por lo tanto $x+u \in G^{l}(E)$, de donde $G^{l}(E)$ es un conjunto abierto en $E$.

Similarmente, podemos demostrar que $G^{r}(E)$ es un conjunto abierto en $E$. Por ser $G(E)=G^{r}(E) \cap G^{l}(E)$, entonces $G(E)$ también es abierto.

iii) Sean $x \in G(E)$ y $\|y\|<\left\|x^{-1}\right\|^{-1}$. Entonces la serie $\sum_{i=0}^{\infty}\left(y x^{-1}\right)^{i}$ es convergente en $E$ y se tiene que $(x-y)^{-1}=x^{-1} \sum_{i=0}^{\infty}\left(y x^{-1}\right)^{i}$. Así

$$
\begin{aligned}
\left\|(x-y)^{-1}-x^{-1}\right\| & =\left\|x^{-1} \sum_{i=1}^{\infty}\left(y x^{-1}\right)^{i}\right\| \leq\left\|x^{-1}\right\| \sum_{i=1}^{\infty}\|y\|^{i}\left\|x^{-1}\right\|^{i} \\
& =\frac{\|y\|\left\|x^{-1}\right\|^{2}}{1-\|y\|\left\|x^{-1}\right\|} .
\end{aligned}
$$

De donde $(x-y)^{-1} \rightarrow x^{-1}$, cuando $\|y\| \rightarrow 0$.

Corolario 1.1.11. Sea E un álgebra de Banach. Entonces:

i) La cerradura de un ideal propio de E también es un ideal propio;

ii) Todo ideal (izquierdo, derecho) maximal es cerrado.

Prueba. i) Sea $I$ ideal propio de $E$. Usando la continuidad de la multiplicación, se prueba fácilmente que la cerradura de $I$ es nuevamente un ideal. Dado que $I \cap G(E)$ es vacío y por Teorema 1.1.10 (ii), se tiene que $I \subset E \backslash G(E)$ con $E \backslash G(E)$ cerrado en $E$. De donde $\bar{I} \cap G(E)$ es vacío. Por lo tanto $\bar{I}$ es un ideal propio de $E$.

ii) inmediata.

Demostración 1.1.12. Sea $E$ un álgebra y $x \in E$. El espectro de $x$ en $E$ es el conjunto de todos los números complejos $\lambda$ tales que $\lambda e-x$ es no invertible en E. El espectro de $x$ en $E$ será denotado por $S p_{E}(x)$ o $S p(x)$, si es claro en qué álgebra se está trabajando.

Si $E$ es un álgebra de Banach y $x \in E$, por el Teorema 1.1.10 (ii), $S p_{E}(x)$ es un subconjunto cerrado de $\mathbb{C}$. La función $\lambda \rightarrow(\lambda e-x)^{-1}$, definida en el conjunto abierto $\mathbb{C} \backslash S p_{E}(x)$, es llamada la resolvente o función resolvente de $x$, denotada por $R(x ; \cdot)$. Al conjunto $\mathbb{C} \backslash S p_{E}(x)=p(x)$ se le llama conjunto resolvente del elemento $x$

Teorema 1.1.13. Sea E un álgebra de Banach. Entonces la función resolvente $\lambda \rightarrow(\lambda e-x)^{-1}$ es analitica en $\mathbb{C} \backslash S p_{E}(x)$. 
Demostración. Para $\lambda_{1}, \lambda_{2} \in \mathbb{C} \backslash S p_{E}(x)$ tenemos que

$$
\begin{aligned}
\left(x-\lambda_{1}\right)^{-1}-\left(x-\lambda_{2}\right)^{-1} & =\left(x-\lambda_{1}\right)^{-1}\left(\left(x-\lambda_{2}\right)-\left(x-\lambda_{1}\right)\right)\left(x-\lambda_{2}\right)^{-1} \\
& =\left(x-\lambda_{1}\right)^{-1}\left(\lambda_{1}-\lambda_{2}\right)\left(x-\lambda_{2}\right)^{-1} \\
& =\left(\lambda_{1}-\lambda_{2}\right)\left(x-\lambda_{1}\right)^{-1}\left(x-\lambda_{2}\right)^{-1} .
\end{aligned}
$$

Así, por el Teorema 10 (iii)

$$
\begin{aligned}
\lim _{\lambda_{1} \rightarrow \lambda_{2}} \frac{\left(x-\lambda_{1}\right)^{-1}-\left(x-\lambda_{2}\right)^{-1}}{\lambda_{1}-\lambda_{2}} & =\lim _{\lambda_{1} \rightarrow \lambda_{2}} \frac{\left(\lambda_{1}-\lambda_{2}\right)\left(x-\lambda_{1}\right)^{-1}\left(x-\lambda_{2}\right)^{-1}}{\lambda_{1}-\lambda_{2}} \\
& =\lim _{\lambda_{1} \rightarrow \lambda_{2}}\left(x-\lambda_{1}\right)^{-1}\left(x-\lambda_{2}\right)^{-1}=\left(x-\lambda_{2}\right)^{-2} .
\end{aligned}
$$

Por lo tanto, la función resolvente es analítica en $\mathbb{C} \backslash S p_{E}(x)$.

El siguiente resultado es uno de los más importantes en la teoría de álgebras de Banach, el cual da origen al importante Teorema de Gelfand-Mazur.

Teorema 1.1.14. Sean $E$ un álgebra de Banach y $x \in E$. Entonces el espectro de $x, S p_{E}(x)$, es un subconjunto compacto no vacío de $\mathbb{C}$.

Demostración. Sea $|\lambda|>\|x\|$. Dado que $\lambda e-x=\lambda\left(e-\lambda^{-1} x\right)$ y $\left\|\lambda^{-1} x\right\|$ $<1$, entonces $e-\lambda^{-1} x \in G(E)$; lo cual implica que $\lambda e-x \in G(E)$. Por lo tanto $\lambda \notin S p_{E}(x)$. Así $S p_{E}(x)$ es acotado y, en consecuencia, compacto.

Supongamos que $S p_{E}(x)=\emptyset$. Por el Teorema 1.1.13 la función resolvente $R(x ; \cdot): \mathbb{C} \backslash S p_{E}(x)=\mathbb{C} \rightarrow E$ es una función holomorfa. Además

$$
\|R(x ; \lambda)\|=\left\|(\lambda e-x)^{-1}\right\|=\left|\lambda^{-1}\right|\left\|\left(e-\lambda^{-1} x\right)^{-1}\right\| \rightarrow 0
$$

cuando $|\lambda| \rightarrow \infty$. Así $R(x ; \lambda)$ es acotada y holomorfa. En consecuencia, se tiene que el mapeo $\lambda \rightarrow \varphi(R(x ; \lambda))$ es holomorfo de valor complejo $\forall \varphi \in E^{\prime}$ el cual también es acotado, puesto que $\varphi$ es continua y $R(x ; \lambda)[\mathbb{C}]$ es acotado en $E$. Por el Teorema de Louville, el mapeo $\lambda \rightarrow \varphi(R(x ; \lambda))$ es constante. Pero

$$
\varphi(R(x ; \lambda))=\varphi\left(\lambda^{-1}\left(e-\lambda^{-1} x\right)^{-1}\right) \rightarrow 0
$$

cuando $|\lambda| \rightarrow \infty$. Así

$$
\varphi(R(x ; \lambda))=0 \quad \forall \varphi \in E^{\prime}, \forall \lambda \in \mathbb{C} ;
$$

de donde $R(x ; \lambda)=0 \forall \lambda \in \mathbb{C}$ Por lo tanto $(\lambda e-x)$ no es invertible $\forall \lambda \in \mathbb{C}$, lo cual es una contradicción. Así $S p_{E}(x) \neq \emptyset$.

Observación. Sea $T$ un operador sobre un espacio de Banach $X$ de dimensión finita, es decir $T$ es una matriz cuadrada. Entonces $S p_{E}(T)$ es finito y consiste de eigenvalores de $T$. 
El hecho de ser $S p_{E}(T)$ no vacío es equivalente al "teorema fundamental del álgebra", de que todo polinomio complejo tiene una raíz. Esto ilustra qué tan profundo es el teorema previo, y también que los operadores sobre espacios de dimensión finita están lejos de ser triviales.

Corolario 1.1.15 (Gelfand-Mazur). Sea E un álgebra de Banach tal que todo elemento no cero de $E$ es invertible (es decir, $E$ es un campo). Entonces $E$ consiste de múltiplos escalares de la unidad e, $E=\{\lambda e: \lambda \in \mathbb{C}\}$. Así, E es isométricamente isomorfo al campo de los números complejos.

Demostración. Por el Teorema 1.1.14, el espectro de $x$ en $E$ es no vacío. Por lo tanto existe $\lambda \in S p_{E}(x)$ tal que $x-\lambda e$ no pertenece a $G(E)$. En consecuencia $x=\lambda e$.

Lema 1.1.16. Sean $x, x_{n}, y_{n}(n=1,2,3, \ldots)$ elementos de un álgebra de Banach $E$ tales que $x_{n} \rightarrow x$ y $\sup _{n}\left\{\left\|y_{n}\right\|\right\}<\infty$.

i) Si $y_{n} x_{n}=e$, para toda $n$, entonces $x \in G^{l}(E)$

ii) Si $x_{n} y_{n}=e$, para todo $n$, entonces $x \in G^{r}(E)$.

Demostración. Tomemos $n$ tal que $\left\|x-x_{n}\right\|<\left(\sup \left\|y_{n}\right\|\right)^{-1}$. Entonces

$$
y_{n} x=y_{n} x_{n}+y_{n}\left(x-x_{n}\right)=e+y_{n}\left(x-x_{n}\right),
$$

donde $\left\|y_{n}\left(x-x_{n}\right)\right\|<1$, y así $y_{n} x \in G(E)$. Por lo tanto $\left(y_{n} x\right)^{-1} y_{n} x=e \mathrm{y}$ $x \in G^{l}(E)$.

La segunda afirmación se prueba en forma similar.

Definición 1.1.17. ( $i)$ Un elemento a, diferente de cero, de un álgebra $E$ es llamado un divisor izquierdo (derecho) de cero si ax $=0(x a=0)$ para algún elemento $x$ no cero de $E$.

(ii) Un elemento a, diferente de cero, de un álgebra de Banach E es llamado un divisor topológico izquierdo (derecho) de cero si

$$
\begin{gathered}
\inf \{\|a x\|: x \in E,\|x\|=1\}=0 \\
(\inf \{\|x a\|: x \in E,\|x\|=1\}=0) .
\end{gathered}
$$

$O$, equivalentemente, existe una sucesión $\left\{x_{n}\right\} \subset E$, con $\left\|x_{n}\right\| \geq \varepsilon>0$, tal que

$$
\lim _{n \rightarrow \infty} a x_{n}=0 \quad\left(\lim _{n \rightarrow \infty} x_{n} a=0\right) .
$$

Los divisores topológicos de cero están cercanamente relacionados a los elementos no invertibles.

Teorema 1.1.18. Sean E un álgebra de Banach y $a \in E$. Entonces: 
i) Si a tiene un inverso izquierdo (derecho), entonces a no es un divisor topológico derecho (izquierdo) de cero;

ii) Si a es invertible, entonces a no es divisor topológico derecho e izquierdo de cero;

iii) Si a $\in \partial G^{l}(E)$ (la frontera topológica de $G^{l}(E)$ ), entonces a es un divisor topológico derecho de cero;

iv) Si $a \in \partial G(E)$, entonces a es un divisor topológico izquierdo y derecho de cero.

Demostración. i) Supongamos que $b$ es un inverso izquierdo de $a$ y sea $x \in E$, con $\|x\|=1$. Entonces tenemos que

$$
1=\|x\|=\|e x\|=\|b a x\| \leq\|b\|\|a x\| .
$$

De donde

$$
\inf \{\|a x\|: x \in E,\|x\|=1\} \geq\|b\|^{-1}>0 .
$$

Así, a no es un divisor topológico derecho de cero. En forma similar lo demostramos para cuando $a$ tiene un inverso por la derecha. El inciso (ii) es consecuencia del inciso $(i)$.

(iii) Sea $a \in \partial G^{l}(E)$ entonces existen sucesiones $\left\{a_{n}\right\},\left\{b_{n}\right\}$ en $E$ tales que $\lim _{n \rightarrow \infty} a_{n}=a$ y $b_{n} a_{n}=e$, para todo $n$. Por Lema 1.1.16, $\lim _{n \rightarrow \infty}\left\|b_{n}\right\|=\infty$; en consecuencia la sucesión $\left\{b_{n}\right\}$ es no acotada. Esto implica que existe una subsucesión $\left\{b_{n_{k}}\right\} \subset\left\{b_{n}\right\}$ tal que $\left\|b_{n}\right\| \rightarrow \infty$ cuando $k \rightarrow \infty$. Sea $c_{k}=\frac{b_{n_{k}}}{\left\|b_{n_{k}}\right\|}$. Entonces $\left\|c_{k}\right\|=1$ para todo $k \mathrm{y}$

$$
\left\|c_{k} a\right\|=\left\|\frac{b_{n_{k}}}{\left\|b_{n_{k}}\right\|}\left(a_{n_{k}}+\left(a-a_{n_{k}}\right)\right)\right\| \leq \frac{1}{\left\|b_{n_{k}}\right\|}+\left\|a-a_{n_{k}}\right\| .
$$

En consecuencia $\lim _{k \rightarrow \infty}\left\|c_{k} a\right\|=0$ y a es un divisor topológico derecho de cero.

$i v)$ Sea $a \in \partial G(E)$, entonces existe $\left\{a_{n}\right\} \subset G(E)$ tal que $\lim _{n \rightarrow \infty} a_{n}=a$. Por el Lema 1.1.16, $\lim _{n \rightarrow \infty}\left\|a_{n}^{-1}\right\|=\infty$; en consecuencia la sucesión $\left\{a_{n}^{-1}\right\}$ es no acotada. Esto implica que existe una subsucesión $\left\{a_{n_{k}}^{-1}\right\} \subset\left\{a_{n}^{-1}\right\}$ tal que $\left\|a_{n_{k}}^{-1}\right\| \rightarrow \infty$ cuando $k \rightarrow \infty$. Sea $c_{k}=\frac{a_{n_{k}}^{-1}}{\left\|a_{n_{k}}^{-1}\right\|}$. Entonces $\left\|c_{k}\right\|=1 \mathrm{y}$, como en (iii), podemos obtener fácilmente que $\lim _{k \rightarrow \infty}\left\|c_{k} a\right\|=0 \mathrm{y} \lim _{k \rightarrow \infty}\left\|a c_{n}\right\|=0$. Así, $a$ es un divisor topológico izquierdo y derecho de cero.

\subsection{Conceptos básicos de álgebras de Banach conmutativas}

En esta sección damos un panorama de resultados básicos de la teoría de álgebras de Banach conmutativas. El ejemplo más importante de tales álgebras es el 
álgebra $C(K)$ de todas las funciones continuas de valor complejo definidas sobre un espacio compacto Hausdorff $K$ no vacío, junto con la norma supremo $\|f\|=$ $\sup \{|f(x)|: x \in K\}$.

En álgebras conmutativas, las nociones de ideal izquierdo, ideal derecho e ideal bilateral coinciden. De la misma forma coinciden las nociones de ideal maximal izquierdo e ideal maximal derecho y de divisor topológico derecho e izquierdo de cero.

Así, únicamente hablaremos acerca de ideales, ideales maximales y de divisores topológicos de cero. En toda esta sección las álgebras serán conmutativas.

Definición 1.2.1. Sean $E$ un espacio vectorial complejo y $A \subset E$ un subespacio de $E$. Decimos que $E$ es de codimensión uno si $\operatorname{dim} E / A=1$.

Teorema 1.2.2. Todo ideal maximal en un álgebra de Banach conmutativa es cerrado y de codimensión uno.

Demostración. Sea $I$ un ideal maximal de $E$ álgebra de Banach conmutativa. Por Corolario 1.1.11 (ii) , I es cerrado. Además, E/I es un álgebra de Banach conmutativa cuyos elementos no cero son invertibles. Por el Corolario 1.1 .15

$$
\operatorname{dim} E / I=1
$$

Definición 1.2.3. Sea E un álgebra de Banach conmutativa. Una funcional lineal (no necesariamente continua) $f: E \rightarrow \mathbb{C}$ es llamada multiplicativa si $f(e)=1 \quad y \quad f(x y)=f(x) f(y)$.

Observación. En general, si $E$ no tiene unidad, la condición $f(e)=1$ se sustituye por la condición $f(x) \neq 0$, para algún $x \in E$. Dado que en esta sección estamos trabajando con álgebras conmutativas, ambas condiciones, junto con la condición $f(x y)=f(x) f(y)$, resultan ser equivalentes.

Las funcionales lineales multiplicativas están en correspondencia uno-uno con los ideales maximales.

Teorema 1.2.4. Sea E un álgebra de Banach conmutativa.

i) Si $f$ es una funcional lineal multiplicativa en E, entonces ker $f$ es un ideal maximal.

ii) Si $I \subset E$ es un ideal maximal en $E$, entonces $E=\{x+\lambda e: x \in I, \lambda \in \mathbb{C}\}$ y el mapeo $f: E \rightarrow \mathbb{C}$, definido por $f(x+\lambda e)=\lambda$, es una funcional lineal multiplicativa. Claramente $\operatorname{ker} f=I$.

Demostración. $i$ ) Es claro que ker $f$ es un subespacio vectorial de $E$. Sean $x \in \operatorname{ker} f \quad$ y $y \in E$. Dado que $f(x y)=f(x) f(y)=0$, entonces ker $f$ es un ideal de $E$, el cual es de codimensión uno. Así, ker $f$ es maximal. 
ii) Sea $I \subset E$ un ideal maximal de $E$. Por Corolario 1.1.11 (ii), $I$ es cerrado; en consecuencia $E / I$ es un álgebra de Banach conmutativa cuyos elementos no cero son invertibles. Así, por el Corolario 1.1.15, $E / I$ es isomorfo a $\mathbb{C}$ y el homomorfismo natural $f: E \rightarrow E / I=\mathbb{C}$ define una funcional lineal multiplicativa en $E$ tal que ker $f=I$.

Observación. En el teorema anterior es importante notar que el álgebra de Banach en cuestión sea conmutativa y que tenga unidad. Si alguna de estas dos condiciones no se cumple, el teorema anterior es falso.

a) Sea $E=M_{2 \times 2}(\mathbb{C})$ el álgebra de todas las matrices complejas de $2 \times 2$ con la norma usual. $E$ es un álgebra de Banach no conmutativa cuya unidad es la matriz

$$
\left(\begin{array}{ll}
1 & 0 \\
0 & 1
\end{array}\right)
$$

Consideremos $a_{1}, a_{2} \in E$ definidas por

$$
a_{1}=\left(\begin{array}{cc}
0 & 1 \\
0 & 0
\end{array}\right) \quad a_{2}=\left(\begin{array}{cc}
0 & 0 \\
1 & 0
\end{array}\right),
$$

entonces

$$
a_{1}^{2}=a_{2}^{2}=\left(\begin{array}{cc}
0 & 0 \\
0 & 0
\end{array}\right) \quad a_{1} a_{2}+a_{2} a_{1}=\left(\begin{array}{cc}
1 & 0 \\
0 & 1
\end{array}\right),
$$

lo cual implica que $E$ no puede tener una funcional lineal multiplicativa.

b) Sea $L^{1}(0,1)$ el espacio de Banach de las funciones absolutamente integrables de valor complejo definidas sobre el intervalo $(0,1)$ con la norma

$$
\|f\|=\int_{0}^{1}|f(t)| d t
$$

Este es un álgebra de Banach conmutativa sin unidad si la multiplicación es definida como la convolución; es decir, dados $f, g \in L^{1}(0,1)$ la multiplicación de $f$ y $g$ es definida por

$$
(f * g)(x)=\int_{0}^{1} f(x-t) g(t) d t .
$$

El álgebra $L^{1}(0,1)$ no tiene funcionales lineales multiplicativas. En efecto, sea $f \in L^{1}(0,1)$ y sea $f(t)=0$, para $t \in(0, \varepsilon)$ y para algún $\varepsilon, 0<\varepsilon \leq 1$. Entonces $(f * f * f * \ldots * f)(x)=0$ para $n$ lo suficientemente grande. Sea $F$ funcional lineal multiplicativa en $L^{1}(0,1)$, entonces $F[f * f * f * \ldots * f]=$ $[F(f)]^{n}=F(0)=0$, de donde $F(f)=0$. Pero el conjunto de esas funciones en $L^{1}(0,1)$, que son igual a cero en una vecindad del cero, es denso y como las funcionales lineales son continuas entonces $F$ es la funcional cero. En consecuencia no existen funcionales lineales multiplicativas en $L^{1}(0,1)$. 
El conjunto de todas las funcionales lineales multiplicativas en un álgebra de Banach conmutativa $E$ será denotado por $M(E)$. Como las funcionales lineales multiplicativas están en correspondencia uno a uno con los ideales maximales, las funcionales lineales multiplicativas son frecuentemente identificadas con los correspondientes ideales maximales.

Las funcionales lineales multiplicativas son la herramienta básica en los problemas relacionados con álgebras de Banach conmutativas.

Como los ideales maximales de un álgebra de Banach son cerrados y dado que ellos son, precisamente, los conjuntos donde las funcionales lineales multiplicativas se anulan, entonces tenemos el siguiente

Teorema 1.2.5. Sea E un álgebra de Banach conmutativa. Toda funcional lineal multiplicativa en $E$ es continua.

Observación. En vista del resultado previo y de la definición 1.1.6, toda funcional lineal multiplicativa $f: E \rightarrow \mathbb{C}$, con $E$ álgebra de Banach conmutativa, es un homomorfismo.

Teorema 1.2.6. Sean E un álgebra de Banach conmutativa $y x \in E$. Tenemos lo siguiente:

i) Si $f \in M(E)$ entonces $f(x) \in S p_{E}(x)$;

ii) Si $\lambda \in S p_{E}(x)$ entonces existe $f \in M(E)$ tal que $f(x)=\lambda$;

iii) Un elemento $x \in E$ es invertible si, y sólo si, $f(x) \neq 0$, para todo $f \in M(E)$.

Demostración. i) Si $f \in M(E)$ entonces el elemento $x-f(x) e \in \operatorname{ker} f$. Como el ker $f$ es un ideal propio, el elemento $x-f(x) e$ es no invertible. Por lo tanto $f(x) \in S p_{E}(x)$.

ii) Si $\lambda \in S p(x)_{E}$ entonces $x-\lambda e$ es no invertible en $E$; de donde $x-\lambda e$ está contenido en un ideal propio, y así existe un ideal maximal que contiene a $x-\lambda e$. La correspondiente funcional lineal multiplicativa $f$ satisface que $f(x-\lambda e)=0$, y así $f(x)=\lambda$.

iii) Se sigue de $(i)$ y $(i i)$.

Teorema 1.2.7. Sea E un álgebra de Banach. Entonces $M(E)$ es no vacío.

Demostración. Si $E$ es un campo entonces, por Corolario 1.1.15, $E$ es isomorfo al campo complejo. Así, la identidad es una funcional lineal multiplicativa.

Si $E$ no es un campo, entonces existe $x \in E$ tal que $x \neq 0$ y $x$ es no invertible. Así, el ideal generado por $x, x E$, es propio. Por el Lema de Zorn-Kuratowsky, $x E$ está contenido en un ideal maximal. En consecuencia existe $f \in M(E)$ y $f(x)=0$. 
Definimos una topología (de la convergencia puntual de las funcionales lineales multiplicativas) en $M(E)$. La base de vecindades abiertas de $f \in M(E)$ está formada por los conjuntos

$$
U_{x_{1}, x_{2}, \ldots, x_{n} ; \varepsilon}=\left\{g \in M(E):\left|f\left(x_{i}\right)-g\left(x_{i}\right)\right|<\varepsilon, i=1,2, \ldots, n\right\},
$$

donde $x_{1}, x_{2}, \ldots, x_{n} \in E, \varepsilon>0$. Esta topología, debida a Gelfand, se llama Topología de Gelfand.

El conjunto $M(E)$, dotado con la topología de Gelfand, da el importante

Teorema 1.2.8. Sea $E$ un álgebra de Banach. $M(E)$, con la topología de Gelfand, es un espacio Hausdorff compacto no vacío.

Demostración. Ver [Ż5; pags. 43-44].

Consideremos el álgebra de Banach $C(M(E))$ de todas las funciones continuas de valor complejo definidas en el espacio compacto Hausdorff $M(E)$, con la norma supremo.

Definición 1.2.9. Sea E un álgebra de Banach conmutativa. El mapeo

$$
\text { ^ }: E \rightarrow C(M(E))
$$

definido por

$$
x \rightarrow \widehat{x}(f)=f(x),
$$

con $x \in E, f \in M(E)$, es llamado la transformada de Gelfand.

Definición 1.2.10. Sean E un álgebra de Banach y $x \in E$. El radio espectral de $x$, denotado por $r(x)$, es el número

$$
r(x)=\max \left\{|\lambda|: \lambda \in \operatorname{Sp}_{E}(x)\right\}
$$

o, equivalentemente por ser E conmutativa con unidad,

$$
r(x)=\max _{I \in M(E)}\{|\widehat{x}(I)|\} \leq\|x\| .
$$

Teorema 1.2.11 (fórmula del radio espectral). Sean $E$ un álgebra de Banach $y x \in$ E. Entonces

$$
r(x)=\lim _{n \rightarrow \infty}\left(\left\|x^{n}\right\|\right)^{\frac{1}{n}} .
$$

Demostración. Ver [M2; pag. 8].

Ahora pasemos a la noción del radical de un álgebra de Banach. 
Definición 1.2.12. Sea E un álgebra de Banach. El radical de E, denotado por rad E, es definido como

$$
\operatorname{rad} E=\cap\{I \in M(E)\} .
$$

Observación. El radical es el ideal cerrado más grande contenido en todos los ideales maximales de $E$. También tenemos que

$$
\operatorname{rad} E=\left\{x \in E: \lim _{n \rightarrow \infty}\left(\left\|x^{n}\right\|\right)^{\frac{1}{n}}=0\right\}=\{x \in E: \widehat{x}(I)=0\},
$$

y así rad $E$ es el núcleo de la transformada de Gelfand. Los elementos en rad $E$ son llamados elementos topológicamente nilpotentes. El álgebra $E$ es semisimple si $\mathrm{rad} E=\{0\}$.

La transformada de Gelfand tiene las siguientes propiedades

Teorema 1.2.13. Sea E un álgebra de Banach conmutativa. Entonces:

i) La transformada de Gelfand es un homomorfismo continuo y $\left\|^{\wedge}\right\|=1$;

ii) $\|\widehat{x}\|=r(x), x \in E$;

iii) $\widehat{x}[M(E)]=S p_{E}(x), x \in E$;

iv) $\widehat{x}=0$ si, y sólo si, $\operatorname{Sp}_{E}(x)=\{0\}$ si, y sólo si, $x \in \operatorname{rad} E$.

Demostración. (iii) $\widehat{x}[M(E)]=\{\widehat{x}(f): f \in M(E)\}=\{f(x): f \in M(E)\}=$ $S p(x)$. $r(x)$.

(ii) $\|\widehat{x}\|=\sup _{f \in M(E)}|\widehat{x}(f)|=\sup _{f \in M(E)}|f(x)|=\sup \{|f(x)|: f \in M(E)\}=$

(i) $\|\widehat{x}\|=\sup _{f \in M(E)}|\widehat{x}(f)|=\sup _{f \in M(E)}|f(x)| \leq \sup _{f \in M(E)}\|f\|\|x\|=\sup _{f \in M(E)}\|x\|=$ $\|x\|$.

Así, la transformada de Gelfand es continua y, además, $\left\|^{\wedge}\right\| \leq 1$.

Por otra parte

$$
\|\widehat{e}\|=\sup _{f \in M(E)}|\widehat{e}(f)|=\sup _{f \in M(E)}|f(e)|=\sup _{f \in M(E)} 1=1 .
$$

Así que

$$
\|\wedge\|=1 .
$$

(iv) $\widehat{x}=0$ si, y sólo si, $\widehat{x}(f)=0$ para todo $f \in M(E)$ si, y sólo si, $f(x)=0$ para todo $f \in M(E)$ si, y sólo si, $S p_{E}(x)=\{0\}$. Ahora, un elemento $x$ pertenece al radical de $E$ si, y sólo si, $x$ está contenido en todos los ideales maximales; es decir $f(x)=0$ para todo $f \in M(E)$. Esto significa que $S p_{E}(x)=\{0\}=\operatorname{rad}$ E. 
Definición 1.2.14. Un subconjunto cerrado $F \subset M(E)$ es llamado un conjunto maximizante para un álgebra de Banach E si

$$
\max _{I \in M(E)}|\widehat{x}(I)|=\max _{I \in F}|\widehat{x}(I)|
$$

para cada $x \in E$. Una frontera de Shilov de E, denotada por $\Gamma(E)$, es cualquier conjunto maximizante minimo.

Definición 1.2.15. Un subconjunto $S$ de un álgebra de Banach $E$ consiste de divisores topológicos de cero combinados (abreviado DTC combinados) si

$$
\inf _{\|z\|, z \in E} \sum_{i=1}^{n}\left\|x_{i} z\right\|=0,
$$

para todo subconjunto finito $\left\{x_{1}, \ldots, x_{n}\right\} \subset S$.

Definición 1.2.16. Sea E un álgebra de Banach conmutativa con unidad. El conjunto de todas las funcionales lineales multiplicativas $f \in M(E)$ con ker $f \in ł(E)$ es la corteza de $E$ y se denota por cor $E$. $ł(E)$ es el conjunto de todos los ideales de E que consisten de DTC combinados

La siguiente proposición da otra caracterización de los DTC combinados.

Proposición 1.2.17. Sea $E$ un álgebra de Banach. Un subconjunto no vacio $S \subset E$ consiste de DTC combinados si, y sólo si, existe una sucesión $\left\{z_{n}\right\} \subset E,\left\|z_{n}\right\|=1$, tal que

$$
\lim _{n \rightarrow \infty}\left\|z_{n} x\right\|=0 \quad \forall x \in S .
$$

Demostración. Ver [Ż8; pag. 43] 


\section{Capítulo 2}

\section{La propiedad de la proyección de una familia de ideales en subálgebras de álgebras de Banach}

En este capítulo presentamos el enfoque más apropiado a los problemas estudiados en los artículos [W1],[W2] y [W4], en el caso de álgebras conmutativas con unidad. Además de las considerables simplificaciones de las pruebas, obtenemos una importante generalización de los resultados principales en el caso no conmutativo.

Sea $E$ un álgebra de Banach con unidad $e$ y sea $A$ subálgebra de $E$ con unidad tal que la unidad $e$ de $E$ pertenezca a $A$. Por $I^{l}(A)$ es denotada a la familia de todos los ideales izquierdos propios de $A$. $I_{E}^{l}(A)$ denota a la familia de los ideales izquierdos de $A$ que consisten de elementos no invertibles en $E$. Si $A$ es conmutativo usamos la notación $I(A)$ e $I_{E}(A)$, respectivamente.

Por $R(A)$ se denota a la subálgebra cerrada más pequeña de $E$ que contiene a $A$ y al conjunto $\left\{a^{-1}: a \in A \cap G(E)\right\}$.

Definición 2.0.1. Sean $A$ un álgebra con unidad e y $U$ una familia de ideales izquierdos propios en $A$. Decimos que la familia $U$ tiene la propiedad de la proyección si para todo ideal $I \in U$, para toda $k$-upla $a_{1}, a_{2}, a_{3}, \ldots, a_{k}$ de elementos en $I$, que conmutan mutuamente, y para todo $c \in A$ que conmuta con todos los $a_{i}, 1 \leq i \leq k$, existen $\lambda \in \mathbb{C}$ y $J \in U$ tales que $a_{1}, a_{2}, a_{3}, \ldots, a_{k}, c-\lambda e$ pertenecen a $J$.

Si $E$ es álgebra de Banach Harte [H1], Teorema 4.2, demostró que $I^{l}(E)$ tiene la propiedad de la proyección. 
Los artículos mencionados al inicio de este capítulo están relacionados, explícitamente o no, a el hecho de que para un álgebra de Banach conmutativa $E$ y para cualquier subálgebra $A \subset E$, la familia $I_{E}(A)$ tiene la propiedad de la proyección.

En este capítulo consideramos el mismo problema para $E$ un álgebra de Banach arbitraria con unidad y $A \subset E$ subálgebra con unidad tal que $a A a^{-1} \subset$ $A$, para todo $a \in A \cap G(E)$. La subálgebra $A$ no es cerrada en $E$ y el inverso $a^{-1}$ no necesariamente pertenece a $A$.

En la sección 2.1 obtenemos un hecho puramente algebraico acerca del espectro combinado definido por una familia de ideales izquierdos (derechos) de un álgebra $E$ con unidad. Si $U$ es una familia arbitraria de ideales izquierdos de $E$, introducimos para una $k$ - upla de elementos que conmutan mutuamente $a_{1}, a_{2}, \ldots, a_{k} \in E$ :

$$
S p_{U}\left(a_{1}, \ldots, a_{k}\right)=\left\{\left(\lambda_{1}, \ldots, \lambda_{k}\right) \in \mathbb{C}^{k}: \text { existe } I \in U, a_{i}-\lambda_{i} \in I, 1 \leq i \leq k\right\} .
$$

El Teorema 2.1.1 afirma que $S p_{U}$ satisface la fórmula del mapeo espectral si, y sólo si, la familia $U$ tiene la propiedad de la proyección.

En la Sección 2.2 concluimos que $I_{E}^{l}(A)$ tiene la propiedad de la proyección $\mathrm{y}$ en las secciones restantes presentamos algunas aplicaciones del Teorema 2.2.2.

\subsection{Espectro combinado definido por una fami- lia de ideales}

El siguiente teorema es fuertemente motivado por los métodos usados en [H1]. Para un álgebra dada $E$ denotemos por $I^{l}(E)$ al conjunto de todos los ideales izquierdos propios en $E$.

Teorema 2.1.1 Sea E un álgebra con unidad sobre C. Sea $U \subset I^{l}(E)$ una familia de ideales izquierdos propios que poseen la propiedad de la proyección. Entonces para toda $k$-upla $a_{1}, \ldots a_{k} \in E$ que conmutan mutuamente y para todo mapeo polinomial $P: \mathbb{C}^{k} \rightarrow \mathbb{C}^{m}$

$$
P\left(S p_{U}\left(a_{1}, \ldots, a_{k}\right)\right)=S p_{U}\left(P\left(a_{1}, \ldots, a_{k}\right)\right) .
$$

Demostración. Sea $P=\left(P_{1}, \ldots, P_{m}\right)$ un mapeo polinomial. Para cualquier mapeo polinomial $P_{i}$ de $k$ variables $x=\left(x_{1}, \ldots, x_{k}\right)$ y para $\lambda=\left(\lambda_{1}, \ldots, \lambda_{k}\right)$, podemos escribir

$$
P_{i}(x)-P_{i}(\lambda)=\sum_{j=1}^{k} f_{i j}(x)\left(x_{j}-\lambda_{j}\right),
$$


donde $\operatorname{los} f_{i j}$ son polinomios.

Supóngase que $\left(\lambda_{1}, \ldots, \lambda_{k}\right) \in S p_{U}\left(a_{1}, \ldots, a_{k}\right)$, esto implica que $a_{j}-\lambda_{j} \in$ $I, 1 \leq j \leq k$ para algún $I \in U$. Por la fórmula anterior, $P_{i}(a)-P_{i}(\lambda) \in I$ para $1 \leq i \leq m$. En consecuencia $\left(P_{1}(\lambda), \ldots, P_{m}(\lambda)\right) \in S p_{U}\left(P_{1}(a), \ldots, P_{m}(a)\right)$. Esto prueba la siguiente contención de la fórmula del mapeo espectral

$$
P\left(S p_{U}\left(a_{1}, \ldots, a_{k}\right)\right) \subset S p_{U}\left(P\left(a_{1}, \ldots, a_{k}\right)\right) .
$$

Ahora supongamos que $u=\left(u_{1}, \ldots, u_{m}\right) \in S p_{U}(P(a))$ y sea $J \in U$ un ideal el cual contiene a los elementos $P_{i}(a)-u_{i}, 1 \leq i \leq m$. Consideremos la $k+m-u p l a$ $(P(a)-u, a)$ cuyos elementos conmutan. Aplicando $k$ veces la propiedad de la proyección de la familia $U$, podemos encontrar $\lambda=\left(\lambda_{1}, \ldots, \lambda_{k}\right) \in \mathbb{C}^{k}$ e $I \in U$ tales que todos los elementos $P_{i}(a)-u_{i}, 1 \leq i \leq m$ y $a_{j}-\lambda_{j}, 1 \leq j \leq k$ pertenecen a $I$. En particular $\lambda \in S p_{U}\left(a_{1}, \ldots, a_{k}\right)$.

Para $b_{1}, \ldots, b_{k} \in E$ y $c_{1}, \ldots, c_{m} \in E$, tenemos que

$$
s=\sum_{i=1}^{m} c_{i}\left(P_{i}(a)-u_{i}\right)+\sum_{j=1}^{k} b_{j}\left(a_{j}-\lambda_{j}\right) \in I,
$$

por que $I$ es un ideal izquierdo.

Para un $1 \leq l \leq m$ fijo, tomemos $c_{l}=e, c_{i}=0$ para $i$ diferente de $l, \mathrm{y}$ $b_{j}=-f_{l j}(a)$ para $1 \leq j \leq k$. El valor correspondiente de la suma $s$, la cual pertenece a $I$, es $\left(P_{l}(\lambda)-u_{l}\right) e$. Esto implica que $P_{l}(\lambda)-u_{l}=0$. Esto prueba la fórmula del mapeo espectral

$$
P\left(S p_{U}\left(a_{1}, \ldots, a_{k}\right)\right)=S p_{U}\left(P\left(a_{1}, \ldots, a_{k}\right)\right) .
$$

\subsection{La propiedad de la proyección de $I_{E}^{l}(A)$}

Teorema 2.2.1. Sea $E$ un álgebra de Banach con unidad y sea $A \subset E$ subálgebra con unidad. Supongamos que $a A a^{-1} \subset A$, para toda $a \in A \cap G(E)$. Si $I \in I_{E}^{l}(A)$ y $J$ es el ideal izquierdo cerrado generado por $I$ en $R(A)$, entonces $J \in I_{E}^{l}(R(A))$. En particular $J$ es un ideal propio de $R(A)$.

Demostración. Consideremos el conjunto $F$ de todos los elementos de la forma

$$
F=\left\{b^{-1} a: a \in A, b \in A \cap G(E)\right\} .
$$

Obviamente $F \subset R(A), A \subset F$ y $\left\{b^{-1}: b \in A \cap G(E)\right\} \subset F$. El conjunto $F$ es cerrado con respecto a la multiplicación. En efecto, si $r, s \in A \cap G(E)$ y $a, b \in A$ entonces

$$
r^{-1} a s^{-1} b=r^{-1} s^{-1} \operatorname{sas}^{-1} b=(s r)^{-1} \operatorname{sas}^{-1} b \in F,
$$


por que $s a s^{-1} b \in A$.

$F$ es también un espacio vectorial, puesto que

$$
r^{-1} a+s^{-1} b=(s r)^{-1}\left[s a+(s r) r(s r)^{-1} b\right]
$$

y $s a+(s r) r(s r)^{-1} b \in A$.

En consecuencia $R(A)$ es igual a la cerradura de $F$, es decir

$$
R(A)=\overline{\left\{b^{-1} a: a \in A, b \in A \cap G(E)\right\}} .
$$

El ideal cerrado $J$, generado en $R(A)$ por $I$, es la cerradura de

$$
J_{0}=\left\{s^{-1} a: a \in I, s \in A \cap G(E)\right\} .
$$

El conjunto $J_{0}$ es un ideal que consiste de elementos no invertibles en $E$. Lo mismo es cierto para $J=\overline{J_{0}}$ por que $G(E)$ es abierto en $E$.

Observemos que $F$ es la subálgebra más pequeña de $E$ que contiene a $A$ y es cerrada con respecto a la inversión en $E$, mientras que $R(A)$ es el álgebra cerrada más pequeña con la misma propiedad.

Teorema 2.2.2. Sea $E$ un álgebra de Banach y sea $A \subset E$ una subálgebra de $E$ con unidad tal que $a A a^{-1} \subset A$, para todo $a \in A \cap G(E)$. Entonces La familia $I_{E}^{l}(A)$ tiene la propiedad de la proyección.

Demostración. Supóngase lo contrario. Existen $I \in I_{E}^{l}(A)$ y $a_{1}, a_{2}, \ldots, a_{k}$ $\in I$ tales que, para algún $c \in A$ y para todo $\lambda \in \mathbb{C}$, el elemento

$$
u=\sum_{j=1}^{k} x_{j} a_{j}+x_{k+1}(c-\lambda e),
$$

con $x_{j} \in A, 1 \leq j \leq k+1$, es invertible en $E$. En consecuencia $u$ también es invertible en $R(A)$. Por el Teorema 2.2.1, el ideal generado en $R(A)$ por $a_{1}, a_{2}, \ldots, a_{k}$, es propio, mientras que el ideal en $R(A)$ generado por $a_{1}, a_{2}, \ldots, a_{k}$, $c-\lambda e$ es $R(A)$, para todo $\lambda \in \mathbb{C}$. Este hecho contradice el Teorema 4.2 de Harte en $[\mathrm{H} 1]$.

Obsérvese que no se supone que $A$ sea cerrado en $E$. Obviamente, los Teoremas 2.2.1 y 2.2.2 son también verdaderos para la familia $I_{E}^{r}(A)$ bajo la condición: $a^{-1} A a \subset A$, para toda $a \in A \cap G(E)$. 


\section{$2.3 \quad$ Subespectro asociado a $I_{E}^{l}(A)$}

De acuerdo a la terminología introducida por Żelazko en [Ż7], un subespectro en $E$ es un mapeo que asocia a cualquier $k-u p l a$ de elementos $a_{1}, a_{2}, \ldots, a_{k} \in E$, que conmutan mutuamente, un conjunto compacto $\tau\left(a_{1}, a_{2}, \ldots, a_{k}\right) \subset \mathbb{C}^{k}$ tal que:

(1) $\tau\left(a_{1}, a_{2}, \ldots, a_{k}\right) \subset \prod_{i=1}^{k} S p\left(a_{i}\right)$, donde $S p\left(a_{i}\right)$ es el espectro usual de $a_{i}$ en $E$;

(2) $P\left(\tau\left(a_{1}, a_{2}, \ldots, a_{k}\right)\right)=\tau\left(P\left(a_{1}, a_{2}, \ldots, a_{k}\right)\right)$, para cualquier mapeo polinomial $P: \mathbb{C}^{k} \rightarrow \mathbb{C}^{m}$.

\section{Ejemplos de subespectros}

1. Espectro de Harte. Sea $E$ un álgebra de Banach compleja con unidad. El espectro de un elemento $a \in E, S p_{E}(a)$, es el conjunto de números complejos $\lambda$ para los cuales no es cierto que existen $b$ y $b$ en $E$ que satisfacen

$$
b(a-\lambda)=e=(a-\lambda) b^{\prime}
$$

La definición del "espectro" $S p_{E}\left(a_{1}, \ldots, a_{k}\right)$, para una $k$-upla de elementos de $E$, está basada en (2.1). Escribimos

$$
a \cdot b=a_{1} b_{1}+a_{2} b_{2}+\cdots+a_{k} b_{k},
$$

para las $k$-uplas $a=\left(a_{1}, \ldots, a_{k}\right)$ y $b=\left(b_{1}, \ldots, b_{k}\right)$ de elementos de $E$; si

$$
a \cdot b=1
$$

decimos que $a$ es una interpolación izquierda para $b$ en $E$ y $b$ es una interpolación derecha para $a$ en $E$. El "espectro combinado" $S p_{E}(a)$ de una $k-u p l a$ $a=\left(a_{1}, \ldots, a_{k}\right)$ ahora será un conjunto de $k-$ uplas $\lambda=\left(\lambda_{1}, \ldots, \lambda_{k}\right)$ de números complejos para los cuales no es cierto que la $k-$ upla $a-\lambda=$ $\left(a_{1}-\lambda_{1}, \ldots, a_{k}-\lambda_{k}\right)$ tiene interpolaciones derecha e izquierda en $E$. Esto se expresa formalmente en la siguiente

Definición. (i) El espectro izquierdo $S p_{E}^{l}(a)=S p^{l}(a)$ de una $k$ - upla a con respecto a $E$ es el conjunto de todos los $\lambda \in \mathbb{C}^{k}$ para los cuales $a-\lambda$ no tiene interpolaciones izquierdas en $E$

(ii) El espectro derecho $S p_{E}^{r}(a)=S p^{r}(a)$ de una $k-$ upla a con respecto a $E$ es el conjunto de todos los $\lambda \in \mathbb{C}^{k}$ para los cuales $a-\lambda$ no tiene interpolaciones derechas en $E$

(iii) El espectro combinado $S p_{E}(a)=S p(a)$ de una $k$-upla $a$ en $E$ es la unión de los espectros derecho e izquierdo de $a$. 
El espectro de Harte, (iii), es un subespectro en $E$ de acuerdo a la terminología de Żelazko. Para la prueba de los axiomas ver [H1].

2. El espectro puntual aproximativo es otro ejemplo de un subespectro de acuerdo a la terminología de Żelazko. Para la comprobación de los axiomas ver [SŻ] y [Ż7; pags. 252-253]

Definición 2.3.1. Sean $E$ un álgebra de Banach con unidad, $A \subset E$ una subálgebra de $E$ y $a_{1}, a_{2}, \ldots, a_{k} \in A$ que conmutan mutuamente. Definimos el conjunto

$$
S p_{E}\left(a_{1}, \ldots, a_{k}\right)=\left\{\left(\lambda_{1}, \ldots, \lambda_{k}\right) \in \mathbb{C}^{k}: \exists I \in I_{E}^{l}(A),\left(a_{i}-\lambda_{i} e\right) \in I, 1 \leq i \leq k\right\} .
$$

Afirmación. El conjunto $S p_{E}\left(a_{1}, a_{2}, \ldots, a_{k}\right)$ es compacto y satisface que

$$
S p_{E}\left(a_{1}, a_{2}, \ldots, a_{k}\right) \subset \prod_{i=1}^{k} S p\left(a_{i}\right)
$$

Demostración. Sea $\lambda=\left(\lambda_{1}, \lambda_{2}, \ldots, \lambda_{k}\right) \in S p_{E}\left(a_{1}, a_{2}, \ldots, a_{k}\right)$, demostremos que $\lambda \in \prod_{i=1}^{k} S p\left(a_{i}\right)$. Supongamos que $\lambda$ no pertenece a $\prod_{i=1}^{k} S p\left(a_{i}\right)$, entonces existe $j \in\{1,2, \ldots k\}$ tal que $\lambda_{j}$ no pertenece a $S p_{E}\left(a_{j}\right)$. Esto implica que existe $b_{j} \in E$ tal que $\left(a_{j}-\lambda_{j} e\right) b_{j}=e$. Así, $e \in I=\left\langle a_{1}-\lambda_{1} e, \ldots . ., a_{k}-\lambda_{k} e\right\rangle$, el ideal en $A$ generado por la $k-$ upla $a_{1}-\lambda_{1} e, \ldots, a_{k}-\lambda_{k} e$, en consecuencia $I=A$. De donde, $I$ no está en $I_{E}^{l}(A)$ y $\lambda=\left(\lambda_{1}, \lambda_{2}, \ldots, \lambda_{k}\right)$ no está en $S p_{E}\left(a_{1}, a_{2}, \ldots, a_{k}\right)$, lo cual es una contradicción. Por lo tanto $\lambda \in \prod_{i=1}^{k} S p\left(a_{i}\right)$.

Por ser $E$ álgebra de Banach con unidad y $a_{i} \in E, 1 \leq i \leq k$, entonces $S p_{E}\left(a_{i}\right)$ es un subconjunto compacto de $\mathbb{C}$. En consecuencia $S p_{E}\left(a_{i}\right)$ es acotado. Por lo tanto $S p_{E}\left(a_{1}, a_{2}, \ldots, a_{k}\right)$ es también acotado.

Sea $\lambda \in \mathbb{C} \backslash S p_{E}\left(a_{1}, a_{2}, \ldots, a_{k}\right)$. Entonces existen $b_{1}, b_{2}, \ldots, b_{k} \in E$ tales que

$$
\sum_{i=1}^{k} b_{i}\left(a_{i}-\lambda_{i} e\right)=e
$$

es decir $\left(a_{1}-\lambda_{1} e, a_{2}-\lambda_{2} e, \ldots, a_{k}-\lambda_{k} e\right)$ es invertible en $E^{k}$. Sea $\gamma \in \mathbb{C}^{k}$ tal que $\gamma=\left(\gamma_{1}, \gamma_{2}, \ldots, \gamma_{k}\right)$ esté muy cerca de $\lambda \mathrm{y}$

$$
\sum_{i=1}^{k}\left\|b_{i}\right\|\left|\gamma_{i}-\lambda_{i}\right|<1
$$

entonces

$$
\begin{aligned}
\|e-b(a-\gamma)\| & =\|b(a-\lambda)-b(a-\gamma)\|=\|b(\lambda-\gamma)\| \\
& =\left\|\sum_{i=1}^{k} b_{i}\left(\gamma_{i}-\lambda_{i}\right)\right\| \leq \sum_{i=1}^{k}\left\|b_{i}\right\|\left|\gamma_{i}-\lambda_{i}\right|<1 .
\end{aligned}
$$


Así, $b(a-\gamma)$ es invertible en $E$; de donde la $k-u p l a[b(a-\gamma)]^{-1} b$ es un inverso izquierdo de $a-\gamma$. En consecuencia $\gamma \notin S p_{E}\left(a_{1}, a_{2}, \ldots, a_{k}\right)$, si $\gamma$ está lo suficientemente cerca de $\lambda$. Por lo tanto $\mathbb{C} \backslash S p_{E}\left(a_{1}, a_{2}, \ldots, a_{k}\right)$ es abierto. Así, $S p_{E}\left(a_{1}, a_{2}, \ldots, a_{k}\right)$ es cerrado.

Supongamos que $a A a^{-1} \subset A$, para todo $a \in A \cap G(E)$. La propiedad de la proyección de la familia $I_{E}^{l}(A)$ implica la siguiente propiedad de la proyección de $S p_{E}\left(a_{1}, a_{2}, \ldots, a_{k}\right)$ :

Si $\left(\lambda_{1}, \lambda_{2}, \ldots, \lambda_{k}\right) \in S p_{E}\left(a_{1}, a_{2}, \ldots, a_{k}\right)$ y $c \in A$ conmuta con todos los $a_{i}$, entonces existe $\lambda \in \mathbb{C}$ tal que $\left(\lambda_{1}, \lambda_{2}, \ldots, \lambda_{k}, \lambda\right) \in S p_{E}\left(a_{1}, a_{2}, \ldots, a_{k}, c\right)$.

Por el Teorema 2.1.1 se tiene que para todo mapeo polinomial $P: \mathbb{C}^{k} \rightarrow \mathbb{C}^{m}$

$$
P\left(S p_{E}\left(a_{1}, a_{2}, \ldots, a_{k}\right)\right)=S p_{E}\left(P\left(a_{1}, a_{2}, \ldots, a_{k}\right)\right) .
$$

Resumimos todo lo anterior en el siguiente

Teorema 2.3.2. Si $E$ es un álgebra de Banach con unidad y $A$ es una subálgebra con unidad de $E$ tal que $a A a^{-1} \subset A$, para toda $a \in A \cap G(E)$, entonces $S p_{E}\left(a_{1}, a_{2}, \ldots, a_{k}\right)$ es un subespectro en $A$.

En [W4] este resultado fue probado para álgebras de Banach conmutativas. El mismo artículo da algunas caracterizaciones del subespectro de $A$ generado por sus superálgebras.

\subsection{Caso $A$ conmutativa}

Sean $E$ un álgebra de Banach con unidad y $A$ una subálgebra de $E$ conmutativa con unidad. Si $a \in A \cap G(E)$, entonces $a^{-1}$ conmuta con todos los elementos de $A$ y la condición $a A a^{-1} \subset A$ es satisfecha. El Teorema 2.2.2 tiene consecuencias importantes.

Teorema 2.4.1. Sea A una subálgebra conmutativa con unidad de un álgebra de Banach E. Entonces para todo $I \in I_{E}(A)$ existe $J \in I_{E}(A)$ tal que $J$ es de codimensión uno en A y contiene a $I$.

Demostración. Sea $I \in I_{E}(A)$ y consideremos el conjunto

$$
\widetilde{J}_{I}=\left\{M \in I_{E}(A): I \subset M\right\}
$$

junto con la relación de orden dada por la inclusión. Por el Lema de ZornKuratowsky se tiene que $\widetilde{J}_{I}$ tiene un elemento maximal $J$.

Supongamos que $J$ no es de codimensión uno en $A$. Sea $c \in A$ tal que $c-\lambda e \notin J$, para todo $\lambda \in \mathbb{C}$. Por el Teorema 2.2.2, para cualquier $k-$ upla 
$a_{1}, a_{2}, \ldots, a_{k} \in J$ existe $\lambda \in \mathbb{C}$ tal que el ideal $I_{A}\left(a_{1}, a_{2}, \ldots, a_{k}, c-\lambda e\right)$, generado por $a_{1}, a_{2}, \ldots, a_{k}, c-\lambda e$, pertenece a $I_{E}(A)$. Denotemos por

$$
\delta\left(a_{1}, a_{2}, \ldots, a_{k}\right)=\left\{\lambda \in \mathbb{C}: I_{A}\left(a_{1}, a_{2}, \ldots, a_{k}, c-\lambda e\right) \subset I_{E}(A)\right\} .
$$

Este conjunto es no vacío y acotado por que está contenido en el espectro de $c$ en $E$. También es cerrado por que si $u$ no pertenece a $\delta\left(a_{1}, a_{2}, \ldots, a_{k}\right)$, entonces existen $b_{1}, b_{2}, \ldots, b_{k} \in A$ tales que el elemento $\sum_{i=1}^{k} a_{i} b_{i}+(c-\lambda e) b_{k+1}$ es invertible en $E$. Lo mismo es cierto para $v \in \mathbb{C}$ lo suficientemente cerca de $u$ por que $G(E)$ es abierto. En consecuencia, el complemento de $\delta\left(a_{1}, a_{2}, \ldots, a_{k}\right)$ es abierto.

Por la definición de $\delta\left(a_{1}, a_{2}, \ldots, a_{k}\right)$ se tiene que

$$
\delta\left(a_{1}, \ldots, a_{k}, c_{1}, \ldots, c_{m}\right) \subset \delta\left(a_{1}, a_{2}, \ldots, a_{k}\right) \cap \delta\left(c_{1}, c_{2}, \ldots, c_{m}\right) .
$$

La familia de conjuntos compactos $\left\{\delta\left(a_{1}, a_{2}, \ldots, a_{k}\right): a_{1}, a_{2}, \ldots, a_{k} \in J\right\}$ tiene la propiedad de la intersección finita, en consecuencia estos conjuntos tienen un punto en común. Si $u_{0}$ pertenece a todos los conjuntos $\delta\left(a_{1}, a_{2}, \ldots, a_{k}\right)$, entonces el ideal generado por $J$ y $c-u_{0} e$ pertenece a $\widetilde{J}_{I}$ y es más grande que $J$. Esta contradicción prueba que $J$ es de codimensión uno.

En el caso de E conmutativa, el Teorema 2.4.1 fue probado en [W4]. Como un corolario, inmediatamente obtenemos una generalización del resultado principal de [W2].

Sea $A$ un álgebra topológica conmutativa con unidad y sea $K$ un espacio Hausdorff compacto. Consideremos el álgebra de Banach $C(K)$, ver ejemplo 2 de la definición 1.1.4. Sea $\varphi: A \rightarrow C(K)$ un homomorfismo continuo de álgebras. Sea

$$
S(K)=\{x \in A: \varphi(x) \text { se anula en alguna parte de } K\} .
$$

Denotemos por $F_{K}$ a la familia de ideales $I$ de $A$ tales que $I \subset S(K)$.

Teorema 2.4.2. Para todo $I \in F_{K}$ existe un ideal $J$ de $A$ de codimensión uno el cual también pertenece a $F_{K}$ y contiene a $I$.

Demostración. Denotemos por $B$ al rango de $\varphi$, el cual es una subálgebra del álgebra de Banach $C(K)$. Un elemento $a$ de $A$ pertenece a $S(K)$ si, y sólo si, $\varphi(a)$ no es invertible en $C(K)$. El ideal $\varphi(I)$ de $B$ consiste de elementos no invertibles en $C(K)$. Por el Teorema 2.4.1, $\varphi(I)$ está contenido en un ideal $J$ de codimensión uno en $B$, el cual consiste de funciones que se anulan en alguna parte de $K$. Este ideal es el núcleo de la funcional lineal multiplicativa $f$ en $B$ determinada en forma única por la fórmula $a-f(a) e \in J$. El núcleo de la funcional lineal multiplicativa $f \circ \varphi$, definida en $A$, es el elemento de $F_{K}$ que contiene a $I$ y es de codimensión uno en $A$.

En [St] Słodkowsky demostró el siguiente resultado 
Teorema 2.4.3. Sea $E$ un álgebra de Banach conmutativa con unidad. Si $I \in H(E)$ entonces existe $J \in E(E)$ tal que $I \subset J$.

I $(E)$ es el conjunto de todos los ideales de $E$ que consisten de DTC combinados y $E(E)$ denota al conjunto de todos los elementos de $1(E)$ que son ideales maximales en $E$.

Usando el Teorema 2.4.3 tenemos el siguiente

Lema 2.4.4. Sean $E$ un álgebra de Banach conmutativa con unidad y $x \in E, x \neq 0$, un divisor topológico de cero. Entonces existe $J \in E(E)$ tal que $x \in J$.

Demostración. Sea $x \in E, x \neq 0$, un DTC y consideremos el ideal $x E$ de $E$. Este consiste de DTC combinados. En efecto, sea $S=\left\{y_{1}, \ldots, y_{k}\right\} \subset x E$ finito. Dado que $x$ es un DTC, entonces existe una sucesión $\left\{b_{n}\right\} \subset E$ tal que $\left\|b_{n}\right\|=1, \forall n \geq 1, \mathrm{y} \lim _{n \rightarrow \infty} x b_{n}=0$. Como $y_{i}=x a_{i}, 1 \leq i \leq k$, para algún $a_{i} \in E$ y la operación multiplicación es continua, entonces tenemos que

$$
\lim _{n \rightarrow \infty} y_{i} b_{n}=\lim _{n \rightarrow \infty}\left(x a_{i}\right) b_{n}=a_{i}\left(\lim _{n \rightarrow \infty} x b_{n}\right)=0 .
$$

En consecuencia, el ideal $x E$ consiste de DTC combinados. Por lo tanto $x E \in \mathfrak{H}(E)$. Por el Teorema 2.4.3, existe $J \in E(E)$ tal que $x E \subset J$. Así $x \in$ $x E \subset J$.

Teorema 2.4.5. Sea E un álgebra de Banach conmutativa con unidad. Todo ideal que consiste de divisores topológicos de cero está contenido en un ideal maximal de E que también consiste de divisores topológicos de cero.

Demostración. Por el Lema 2.4.4, todo divisor topológico de cero está contenido en un ideal maximal que consiste de DTC combinados. Los ideales de esta clase forman un subconjunto compacto en el espacio $M(E)$, llamado la corteza de $E$. Un elemento $x \in E$ es un DTC si, y sólo si, $\widehat{x}$ se anula en algun elemento de $\operatorname{cor} E$. Tomando $K=\operatorname{cor} E$ y $\varphi(x)=\widehat{x}$, definida sobre cor $E$, obtenemos el resultado deseado por el Teorema 2.4.2.

Sabemos que $\Gamma(E) \subset$ cor $E$ es un subconjunto compacto de la corteza de $E$. Aplicando el Teorema 2.4.2 para $K=\Gamma(E)$ y $\varphi(a)=\widehat{a}$ restringida a $\Gamma(E)$, obtenemos un resultado nuevo análogo al Teorema 2.4.5.

Primero damos una descripción del conjunto $S(\Gamma(E))$, el cual, por definición, es igual a

$$
\{a \in E: 0 \in \widehat{a}(\Gamma(E))\} .
$$

Sea $r(a)$ el radio espectral de $a \in E$.

Proposición 2.4.6. Un elemento a $\in E$ pertenece a $S(\Gamma(E))$ si, y sólo si, $(C)$ existe $a_{n} \in E$ tal que $r\left(a_{n}\right)=1$ y $\lim _{n \rightarrow \infty} r\left(a a_{n}\right)=0$. 
Demostración. Si $a \in E$ y $\widehat{a}$ se anula en alguna parte de $\Gamma(E)$, entonces $a \in I$, para algún $I \in \Gamma(E)$. Fue demostrado en [Ż2] que la condición $(C)$ es válida.

Supongamos ahora que la condición $(C)$ es satisfecha. Dado que

$$
r(a)=\sup _{M(E)}|\widehat{a}|=\sup _{\Gamma(E)}|\widehat{a}|,
$$

se sigue por $(C)$ que $\widehat{a}$ restringido a $\Gamma(E)$ es un divisor topológoco de cero en el álgebra de Banach $C(\Gamma(E))$. En consecuencia, $\widehat{a}$ se anula en alguna parte de $\Gamma(E)$, la cual también es la frontera de Shilov de $C(\Gamma(E))$.

El Teorema 2.4.2 conduce al

Teorema 2.4.7. Sea E un álgebra de Banach conmutativa. Si I es un ideal cuyos elementos satisfacen la condición $(C)$, entonces I está contenido en algún $J \in M(E)$ tal que los elementos de $J$ también satisfacen la condición $(C)$.

\subsection{Un teorema de separación}

Sea $X$ un espacio topológico Hausdorff y sea $A$ una subálgebra con unidad de $C(X)$ tal que las funcionales evaluación $\delta_{x}: f \rightarrow f(x), x \in X$, agotan al conjunto de funcionales multiplicativas de $A$.

Para un conjunto compacto $K \subset X$ el casco $A$-racionalmente convexo de $K$ es definido como sigue:

$$
h(K)=\{x \in X: \forall f \in A, f(x)=0 \text { implica que } 0 \in f(K)\} .
$$

Para una $k$ - upla fija $f_{1}, \ldots, f_{k} \in A$ consideramos

$$
V=\left\{x \in X: f_{1}(x)=\cdots=f_{k}(x)=0\right\} .
$$

Teorema 2.5.1. $V \cap h(K)=\emptyset$ si, y sólo si, existe $f \in A$ tal que $f$ restringida a $V$ es cero y $f$ no se anula en $K$

Demostración. Si la última condición es satisfecha entonces, obviamente, ningún punto de $V$ pertenece a $h(k)$.

Supongamos que esta condición no es satisfecha. Toda $f \in A$, la cual se anula en $V$, alcanza el cero en $K$. Denotemos por $A_{1}$ al álgebra que consiste de las restricciones a $K$ de los elementos de $A$. Sea $I$ el ideal generado en $A_{1}$ por las restricciones de las funciones $f_{j}, 1 \leq j \leq k$. Por nuestra suposición, todo elemento de $I$ se anula en alguna parte de $K$, en consecuencia este no es invertible en $C(K)$. Por Teorema 2.3.2, existe en $A_{1}$ un ideal $J$ de codimensión uno el cual contiene a $I$ y consiste de funciones que alcanzan cero en $K$. El ideal 
$J$ es el núcleo de una funcional lineal multiplicativa $\varphi$ en $A_{1}$. La funcional de $A$ en $\mathbb{C}$, definida por $f \rightarrow \varphi(f \mid V)$, es una funcional lineal multiplicativa, asi que existe $x_{0} \in X$ tal que $\varphi(f \mid V)=f\left(x_{0}\right)$. El ideal $J$ consiste de las restricciones a $K$ de todas las funciones $f \in A$ que se anulan en $x_{0}$. Sabemos que estas funciones satisfacen que $0 \in f(K)$, en consecuencia $x_{0} \in h(K)$. La inclusión $I \subset J$ significa que, en particular, $f_{1}\left(x_{0}\right)=\cdots=f_{k}\left(x_{0}\right)=0$. En consecuencia $x_{0} \in V \cap h(K)$. Así, la demostración se sigue.

Ejemplo. Sea $X=\mathbb{C}^{n}$ y $A=\mathbf{P}(n)$, donde $\mathbf{P}(n)$ denota al álgebra de las funciones polinomiales en $\mathbb{C}^{n}$. Para $\mathbf{p}=\left(p_{1}, \ldots, p_{k}\right) \in \mathbf{P}^{k}$ sea

$$
V=\left\{z \in \mathbb{C}^{n}: p_{1}(z)=\cdots=p_{k}(z)=0\right\}
$$

Si $K$ es un subconjunto compacto de $\mathbb{C}^{n}$, sea

$$
\begin{aligned}
h(K) & =\left\{z \in \mathbb{C}^{n}:|f(z)| \leq \sup _{w \in K}|f(w)|\right\} \\
& =\left\{z \in \mathbb{C}^{n}: \forall f \in \mathbf{P}(n) \quad f(z)=0 \rightarrow 0 \in f(K)\right\},
\end{aligned}
$$

para esta última igualdad ver [Ga; pag. 69]. Entonces tenemos que

$$
V \cap h(K) \neq \emptyset \text { si, y sólo si, }(f \mid V=0 \rightarrow 0 \in f(K), \forall f \in \mathbf{P}(n)) \text {. }
$$




\section{Capítulo 3}

\section{Conceptos básicos de álgebras topológicas}

En este capítulo presentamos definiciones, conceptos y resultados básicos de álgebras topológicas, los cuales serán usados en los capítulos siguientes con el fin de obtener generalizaciones de los resultados demostrados en el capítulo 2 . Para una lectura más amplia de álgebras topológicas ver [Ma],,[Mi],[Ż3].

\subsection{Conceptos básicos}

Definición 3.1.1. Sea $E$ un álgebra. Un subconjunto $U$ de $E$ es llamado absolutamente convexo si éste es balanceado y convexo.

Definición 3.1.2. Sea $E$ un álgebra. Un subconjunto $U$ de $E$ se dice ser multiplicativo si $U U \subset U$ (abreviado $m$ - conjunto).

Definición 3.1.3. Sea $E$ un álgebra. Un subconjunto $U$ absolutamente convexo, absorbente y multiplicativo se llama barril algebraico (abreviado abarril).

Definición 3.1.4. Por un álgebra topológica queremos decir un álgebra E la cual es un espacio vectorial topológico de tal manera que la multiplicación en $E$ sea separadamente continua (es decir, continua en cada una de las variables). Si el espacio vectorial topológico es, en particular, localmente convexo, hablaremos de un álgebra (topológica) localmente convexa. Si la multiplicación de un álgebra topológica $E$ es continua en ambas variables (es decir, conjuntamente continua) 
hablaremos de un álgebra topológica (localmente convexa) con una multiplicación continua.

El siguiente es un hecho estandard en la teoría de los espacios vectoriales topológicos; el resultado análogo en nuestro caso también es útil.

Proposición 3.1.5. Sea E un álgebra topológica y sea $\rho$ una semi-norma (submultiplicativa) sobre E. Entonces las siguientes afirmaciones son equivalentes:

i) $\quad \rho$ es continua en $0 \in E$.

ii) $\rho$ es uniformemente continua en $E$.

iii) $\rho$ es continua en $E$.

iv) El conjunto

$$
A=\{x \in E: \rho(x)<1\}
$$

es abierto en E (llamamos a tal conjunto semi-bola unitaria abierta de $\rho$ ). Además la cerradura de (3.1) es

$$
\bar{A}=\{x \in E: \rho(x) \leq 1\}
$$

(llamamos a tal conjunto la semi-bola unitaria cerrada de $\rho$ ). Finalmente el interior de (3.2) es (3.1).

Los conjuntos de la forma (3.2) juegan un papel fundamental en lo siguiente, en consecuencia distinguimos a ellos.

Definición 3.1.6. Sea $E$ un álgebra topológica. Un subconjunto $U$ de $E$ se dice ser un $m$ - barril si es absolutamente convexo, absorbente, cerrado y multiplicativo.

Definición 3.1.7. Por un álgebra localmente y multiplicativamente convexa E (abreviada álgebra localmente $m$-convexa) queremos decir un álgebra la cuál es, en particular, un espacio vectorial topológico con una base local que consiste de conjuntos $m$ - convexos ( es decir, conjuntos multiplicativos y convexos).

A pesar de que la definición precedente es bastante general, motivada por su utilidad en ejemplos concretos, es realmente mucho más fuerte de lo que parece, como llegará ha ser más claro en lo sucesivo. Así, antes de que tengamos a nuestra disposición la terminología necesaria para ver su total fortaleza, tenemos la siguiente

Proposición 3.1.8. Sea E un álgebra localmente $m$-convexa. Entonces $E$ es un álgebra topológica localmente convexa con la multiplicación continua. 
Demostración. Si $\beta=\{U\}$ es una base local del respectivo espacio vectorial toplógico $E$, consistente de conjuntos m-convexos, entonces $E$ es, en particular, un espacio localmente convexo. Por otra parte, la relación $U U \subset U$, para todo $U \in \beta$, implica que la multiplicación en $E$, cuando es considerada como un mapeo bilineal $E$-valuado sobre $E \times E$, es continua en el origen de $E \times E$, y en consecuencia continua en todo $E \times E$.

Lema 3.1.9. Sea $E$ un álgebra topológica. Entonces para todo $A, B$ subconjuntos de $\mathrm{E}$ se tiene la relación

$$
\bar{A} \bar{B} \subset \overline{A B} .
$$

En particular, si $A, B$ y $C$ son subconjuntos de $E$, entonces

$$
A B \subset C \text { implica que } \bar{A} \bar{B} \subset \bar{C} \text {. }
$$

Asi que si $U$ es un $m$ - conjunto de $E$, su cerradura $\bar{U}$ también es un $m$ - conjunto de E, es decir, se tiene que

$$
\bar{U} \bar{U} \subset \bar{U} .
$$

Demostración. Sean $x \in \bar{A}, y \in \bar{B}$, por demostrar que $x y \in \overline{A B}$ si, y sólo si, $x y$ es punto de acumulación de $A B$ si, y sólo si, $\exists\left\{x_{\alpha} y_{\alpha}\right\}_{\alpha \in \Lambda} \subset A B$, red de $A B$, tal que

$$
x y=\lim _{\alpha} x_{\alpha} y_{\alpha} .
$$

Como $x \in \bar{A}, y \in \bar{B}$, entonces existen $\left\{x_{\alpha}\right\}$ y $\left\{y_{\beta}\right\}$ redes en $A$ y en $B$, respectivamente, tales que $x_{\alpha} \rightarrow x$ y $y_{\beta} \rightarrow y$. Por ser la multiplicación continua tenemos que

$$
x y=\left(\lim _{\alpha} x_{\alpha}\right) y=\lim _{\alpha}\left(x_{\alpha} y\right)=\lim _{\alpha}\left(x_{\alpha}\left(\lim _{\beta} y_{\beta}\right)\right)=\lim _{\alpha}\left(\lim _{\beta}\left(x_{\alpha} y_{\beta}\right)\right) .
$$

Como $x_{\alpha} y_{\beta} \in A B$ entonces $\lim _{\beta}\left(x_{\alpha} y_{\beta}\right) \in \overline{A B}$ para toda $\alpha$. Esto implica que $\lim _{\alpha}\left(\lim _{\beta}\left(x_{\alpha} y_{\beta}\right)\right) \in \overline{A B}$. Así $x y \in \overline{A B}$. Por lo tanto $\bar{A} \bar{B} \subset \overline{A B}$.

Una consecuencia útil del lema previo es el siguiente

Corolario 3.1.10. Sea $E$ un álgebra topológica y $F$ (respectivamente I) una subálgebra (respectivamente un ideal) de E. Entonces la cerradura $\bar{F}$ (respectivamente $\bar{I}$ ) es una subálgebra cerrada (respectivamente ideal cerrado) del álgebra E.

Demostración. Veamos que $\bar{I}$ es un ideal, es decir que $\bar{I}$ es un subgrupo aditivo de $E$ y que $E \bar{I} \subset \bar{I}, \bar{I} E \subset \bar{I}$. Claramente $\bar{I}$ es un subgrupo aditivo puesto que la operación suma es continua. Dado que $E \subset \bar{E}$ entonces

$$
E \bar{I} \subset \bar{E} \bar{I} \subset \overline{E I} \subset \bar{I} .
$$


Similarmente se tiene que $\bar{I} E \subset \bar{I}$.

Como $E$ es un álgebra topológica, entonces $E$ es un espacio vectorial topológico y la multiplicación es continua. Así $\bar{F}$ es un álgebra cerrada en $E$.

El siguiente resultado caracteriza a las álgebras localmente $m$ - convexas.

Teorema 3.1.11. Sea E un álgebra. Entonces las siguientes afirmaciones son equivalentes:

1) E es un álgebra localmente $m$ - convexa (Definición 3.1.7);

2) E es un álgebra topológica con una base local que consiste de $m$-barriles;

3) Existe un filtro base en E que consiste de a-barriles.

Demostración. Ver [Ma; pag 18].

Corolario 3.1.12. Sea $E$ un álgebra. $E$ es un álgebra localmente $m-$ convexa sí, y solo sí, el respectivo espacio vectorial $E$ es un espacio localmente convexo con una base local que consiste de $m$-conjuntos.

Demostración. $\Longrightarrow$ ) Inmediato.

$(\Longleftarrow$ Basta que demostremos que en $E$ existe una base de filtro que consiste de $a$-barriles. Sea $\beta$ base local que consiste de conjuntos $m-$ convexos (es decir, conjuntos multiplicativos y convexos). Sea $(U)=\bigcup_{|\lambda| \leq 1} \lambda U$, claramente este conjunto es balanceado y multiplicativo. Sea $\widetilde{\beta}=\{\langle(U)\rangle: U \in \beta\}$, donde $\langle(U)\rangle$ es el casco convexo de $(U)$. Como $(U)$ es balanceado y multiplicativo, entonces $\langle(U)\rangle$ es también un conjunto multiplicativo, balanceado y convexo de $E$. En consecuencia todo elemento de $\widetilde{\beta}$ es balanceado, absorbente, convexo y multiplicativo. Así, $\widetilde{\beta}$ es una base de filtro en $E$. Por Teorema 3.1.11, $E$ es un álgebra localmente $m$ - convexa.

Es en la forma del Teorema 3.1.11, inciso (2), que la noción de un álgebra localmente $m$-convexa es usualmente aplicada (en conexión con la Proposición 3.1.8). Por otra parte, también sucede que la forma anterior del Teorema 3.1.11 es tomada como definición de un álgebra localmente $m$ - convexa, en particular cuando la topología del álgebra es definida en términos de una familia de semi-normas (submultiplicativas), como lo veremos más adelante. Sin embargo, la definición 3.1.7 o, en particular, el Corolario 3.1.12 son, desde luego, las herramientas más convenientes para ver si un álgebra dada es localmente $m$ - convexa.

No cualquier $m$ - barril en un álgebra topológica, igual en un álgebra localmente $m$-convexa, es una vecindad del cero (ver ejemplo 3.2.2).

Sin embargo, las álgebras topológicas que tienen esta propiedad juegan un papel importante. A continuación consideramos formalmente estas álgebras. 
Definición 3.1.13. Por un álgebra $m$-barrilada queremos decir un álgebra topológica donde todo $m$ - barril es una vecindad del cero.

Un álgebra topológica $m$ - barrilada la cual es, en particular, localmente convexa (localmente $m$ - convexa, respectivamente), será llamada un álgebra localmente $m$-barrilada (álgebra localmente $m$-barrilada $m$-convexa, respectivamente). Toda álgebra topológica para la cual el espacio vectorial topológico respectivo es barrilado se llama álgebra barrilada. Obviamente toda álgebra $m$ - barrilada es un álgebra barrilada, puesto que todo $m$ - barril es un barril (es decir, un conjunto absorbente, convexo,balanceado y cerrado). El caso particular de un álgebra localmente convexa barrilada (localmente $m$-convexa, respectivamente) es claro.

En particular se tiene la situación anterior considerando un álgebra topológica de Baire; esto es, un álgebra topológica para la cual el respectivo espacio vectorial topológico es un espacio de Baire, puesto que este último es un espacio barrilado (ver, por ejemplo, [Tr;pag. 346]).

Por otra parte, considerando un ejemplo más particular, pero importante, de lo anterior llegamos a la siguiente.

Definición 3.1.14. Por un álgebra de Fréchet queremos decir un álgebra topológica para la cual el respectivo espacio vectorial topológico es de Fréchet (es decir, metrizable y completo).

Ciertamente, todo espacio de Fréchet es un espacio de Baire, en consecuencia un espacio barrilado, así toda álgebra de Fréchet es un álgebra barrilada y, a fortiori, un álgebra $m$ - barrilada. En este asunto, también tenemos el ejemplo particular de un álgebra de Fréchet localmente convexa o, más particularmente, el de un álgebra de Fréchet localmente $m$ - convexa.

\subsection{Ejemplos de álgebras topológicas}

1. Sea $E$ un espacio vectorial topológico y sea $L(E)$ el espacio vectorial de los mapeos lineales continuos de $E$ en si mismo. Este espacio equipado con la "topología de convergencia simple" en $E$, llega a ser un espacio vectorial topológico, el cual se denota por $L_{s}(E)$ (ver [Bou, Capítulo 1; pag. 16]). Por otra parte, es fácil de checar que la "composición de mapeos lineales" define en el último espacio una multiplicación separadamente continua. En consecuencia $L_{s}(E)$ es un álgebra topológica. En particular, si $E$ es localmente convexo, entonces $L_{s}(E)$ es un álgebra localmente convexa, dado que su topología puede ser considerada como la "topología inicial" en $L(E)$ definida por los "mapeos evaluación"

$$
\widehat{x}: £(E) \rightarrow E
$$




$$
x \rightarrow \widehat{x}(f)=f(x)
$$

para todo $f \in L(E)$.

2. Sea $B(\mathbb{R})$ el álgebra de las funciones continuas acotadas de valor complejo definidas en $\mathbb{R}$. El último conjunto dotado con la topología de convergencia compacta en $\mathbb{R}$ lo convierte en un álgebra topológica, denotada por $B_{c}(\mathbb{R})$. Efectivamente, considerando los conjuntos

$$
V_{K, \varepsilon}=\{f \in B(\mathbb{R}):|f(x)| \leq \varepsilon, \text { para cualquier } x \in K\},
$$

donde $K$ varia sobre los intervalos compactos de $\mathbb{R}$ y $0<\varepsilon \leq 1$, fácilmente se verifica que la familia (3.5) constituye una base de filtro sobre el álgebra $B(\mathbb{R})$ que consiste de $a$-barriles, así que $B(\mathbb{R})$, topologizado por la familia (3.5), es un álgebra localmente $m$ - convexa (Teorema 3.1.11). Además, la última topología en $B(\mathbb{R})$ es, en efecto, idéntica con la topología $c$ de convergencia compacta en $\mathbb{R}$ como sigue por las mismas definiciones y por

$$
T(K, \varepsilon)=\{f \in B(\mathbb{R}):|f(x)|<\varepsilon, \text { para cualquier } x \in K\} \subset V_{K, \varepsilon},
$$

donde $K$ y $\varepsilon$ varian como en (3.5). En consecuencia, $B_{c}(\mathbb{R})$ es un álgebra localmente $m$ - convexa. Por otra parte, considerando en (3.5) los $K$ variando sobre los intervalos $[-n, n]$ de $\mathbb{R}$, y $\varepsilon$ sobre $\frac{1}{n}$, con $n \in \mathbb{N}$, se obtiene que $B_{c}(\mathbb{R})$ es un álgebra localmente $m$ - convexa metrizable que no es $m$-barrilada, así que a fortiori no es barrilada. La última observación es fácilmente verificada observando que el conjunto

$$
B_{0}(1)=\{f \in B(\mathbb{R}):|f(x)| \leq 1, \text { para cualquier } x \in \mathbb{R}\}
$$

es un $m$-barril en $B_{c}(\mathbb{R})$, el cual no es una vecindad del cero. Así, la topología de convergencia uniforme en $\mathbb{R}$, bajo la cual $B(\mathbb{R})$ es un álgebra de Banach, es diferente de la topología de convergencia compacta en $\mathbb{R}$, topologizando la última álgebra como antes.

3. La mayoria de las afirmaciones enunciadas abajo son demostradas en [Ar]. El espacio (3.7), considerado abajo, puede ser tratado, más naturalmente, como un "límite proyectivo" el cual también es un álgebra apropiadamente topologizada. Su aparición en este momento contribuye, sin embargo, a la clarificación de la definición 3.1.14 en relación a las varias clases de álgebras topológicas bajo discusión. Consideremos el siguiente conjunto

$$
L^{\omega}([0,1])=\bigcap_{p \geq 1} L^{p}([0,1]),
$$

de todas las funciones complejo-valuadas definidas sobre el intervalo unitario cerrado $[0,1] \subset \mathbb{R}$, las cuales son $p$-integrables, para todo $p \geq 1$, con respecto a la medida de Lebesgue sobre $[0,1]$. Este conjunto es, ciertamente, un espacio 
vectorial sobre los complejos, puesto que cada uno de los $L^{p}$ lo es; además, es un álgebra, debido a la relación

$$
\|f g\|_{p} \leq\|f\|_{q}\|g\|_{r},
$$

con $f, g \in L^{\omega}$, y $q, r$ reales positivos tales que $\frac{1}{p}=\frac{1}{q}+\frac{1}{r}$; aquí se define la respectiva $L^{p}$ - norma por la relación

$$
\|f\|_{p}=\left\{\int_{0}^{1}|f(x)|^{p} d x\right\}^{\frac{1}{p}}
$$

para todo $f \in L^{p}([0,1])$ y $p \geq 1$. Por otra parte, los conjuntos

$$
U_{p}(\varepsilon)=\left\{f \in L^{p}:\|f\|_{p}<\varepsilon\right\},
$$

con $p \geq 1$ y $\varepsilon>0$, define una base local de $L^{\omega}$ que consiste de conjuntos convexos. Ahora, dado que el espacio de medida involucrado es acotado, podemos restringirnos a un subconjunto numerable de ṕs en (3.9), obteniendo así una base local numerable de $L^{\omega}$; así que el último espacio se convierte en un espacio localmente convexo metrizable y dado que también es completo, $L^{\omega}$ es un álgebra de Fréchet localmente convexa (con la operación de multiplicación continua). Además, $L^{\omega}$ no puede ser topologizado como un álgebra localmente $m$ - convexa; en efecto, un conjunto $m$ - convexo $U$ en $L^{\omega}$ es una vecindad abierta de cero si, y sólo si,

$$
U=L^{\omega} .
$$

En particular, $L^{\omega}$ no puede ser un álgebra normada. Por otra parte, $L^{\infty}([0,1])$, siendo un álgebra de Banach en su propia norma, es una subálgebra densa de $L^{\omega}$, mientras que $L^{\omega}$ es denso en cada uno de los espacios $L^{p}, p \geq 1$, todos los mapeos inclusión canónicos son continuos.

4. Sea $\alpha$ un entero no negativo. Con cada $\alpha$ asociamos el operador diferencial

$$
D^{\alpha}=\left(\frac{\partial}{\partial x}\right)^{\alpha} \text {. }
$$

Si $\alpha=0, D^{\alpha} f=f$. Decimos que una función $f:[0,1] \rightarrow \mathbb{C}$ pertenece a $C^{\infty}([0,1])$ si $D^{\alpha} f \in C([0,1]), \forall \alpha \geq 0 . C^{\infty}([0,1])$ es un álgebra conmutativa con unidad bajo las operaciones de suma y multiplicación usuales.

Definamos una topología en $C^{\infty}([0,1])$ la cual hace a $C^{\infty}([0,1])$ un álgebra $m$ - convexa de Fréchet, $Q$-algebra y no es normada.

Se define la familia de semi-normas $\left\{\widetilde{\rho}_{N}\right\}$ en $C^{\infty}([0,1]), N=1,2,3, \ldots$, por

$$
\widetilde{\rho}_{N}(f)=\max \left\{\left|D^{\alpha} f(x)\right|: \alpha \leq N, 0 \leq x \leq 1\right\} .
$$

$\left(C^{\infty},\left\{\widetilde{\rho}_{N}\right\}\right)$ es un espacio vectorial topológico localmente convexo de Fréchet. Reemplazamos esta familia de semi-normas por una familia de seminormas equivelente dada por

$$
\rho_{N}(f)=2^{N} \max _{\alpha \leq N} 2^{\alpha} \max _{0 \leq x \leq 1}\left|D^{\alpha} f(x)\right| .
$$


Dado que

$$
\max _{0 \leq x \leq 1}\left|D^{\alpha} f(x)\right| \leq \frac{\rho_{N}(f)}{2^{N+\alpha}},
$$

entonces tenemos que

$$
\begin{aligned}
\rho_{N}(f g) & =2^{N} \max _{\alpha \leq N} 2^{\alpha} \max _{0 \leq x \leq 1}\left|D^{\alpha}(f g)(x)\right| \\
& =2^{N} \max _{\alpha \leq N} 2^{\alpha} \max _{0 \leq x \leq 1}\left|\sum_{k=0}^{\alpha} k_{k}^{\alpha} D^{K} f(x) D^{\alpha-K} g(x)\right| \\
& \leq 2^{N} \max _{\alpha \leq N} 2^{\alpha} \sum_{k=0}^{\alpha} k \max _{0 \leq x \leq 1}\left|D^{K} f(x)\right|\left|D^{\alpha-K} g(x)\right| \\
& \leq 2^{N} \max _{\alpha \leq N} 2^{\alpha} \sum_{k=0}^{\alpha} k\left(\frac{\rho_{N}(f)}{2^{N+\alpha}}\right)\left(\frac{\rho_{N}(g)}{2^{N+\alpha}}\right) \\
& =\rho_{N}(f) \rho_{N}(g) .
\end{aligned}
$$

Así, $C^{\infty}([0,1])$ resulta ser un álgebra de Fréchet localmente $m$ - convexa. También es una $Q$-álgebra, es decir $G\left(C^{\infty}([0,1])\right)$ es un subconjunto abierto de $C^{\infty}([0,1])$. Puede ser demostrado que no hay una topología que haga a $C^{\infty}([0,1])$ un álgebra de Banach (ver [Ru; pags. 33-34].

\subsection{Topologías definidas por semi-normas sub- multiplicativas}

Sea $E$ un álgebra y $\rho$ una semi-norma submultiplicativa en $E$. La semi-bola unitaria de $E$, correspondiente a $\rho$, es el conjunto

$$
U_{\rho}(1)=\{x \in E: \rho(x) \leq 1\} .
$$

Por otra parte, se tiene que

$$
U_{\rho}(\varepsilon)=\{x \in E: \rho(x) \leq \varepsilon\}=\varepsilon U_{\rho}(1),
$$

para todo número real $\varepsilon>0$. En particular el conjunto (3.10) es un $a-b a r r i l$ del álgebra dada $E$. Así, la familia

$$
\beta=\left\{U_{\rho}\left(\frac{1}{n}\right): n \in \mathbb{N}\right\}
$$

da una base de filtro en el álgebra $E$ que consiste de $a$-barriles. En consecuencia, por el Teorema 3.1.11, $E$ es un álgebra localmente $m$-convexa que tiene a $\beta$ como una base local que consiste de $a$-barriles. 
Un par $(E, \rho)$ que consiste de un álgebra $E$ y una semi-norma submultiplicativa $\rho$ definida en $E$ es llamado un álgebra semi-normada, mientras $E$ es considerado así para ser topologizado como antes. Así, se concluye que, por (3.1.11), toda álgebra semi-normada $(E, \rho)$ es un álgebra localmente $m$-convexa metrizable. Además, un álgebra topológica $E$ cuya topología es definida por una semi-norma submultiplicativa es llamada un álgebra semi-normable.

Ahora, sobre la base de la situación que se tiene en el caso de espacios vectoriales topológicos, se concluye que un álgebra semi-normada (semi-normable, respectivamente) $E$ es Hausdorff si, y sólo si, la semi-norma (submultiplicativa) $\rho$, la cual define la topología de $E$, es llamada una norma; es decir, se tiene que $\rho(x)>0 \forall x \neq 0, x \in E$. El par $(E, \rho)$ es entonces llamado un álgebra normada (normable, respectivamente).

Una razón particular de considerar álgebras semi-normadas es debido, desde luego, al hecho de que estas constituyen, en efecto, las bases del edificio para definir un álgebra localmente $m$ - convexa en términos de semi-normas submultiplicativas, como será visto en lo siguiente. Pero aún tenemos que comentar sobre algún material preliminar.

Definición 3.3.1. Sean $E$ y $F$ dos álgebras sobre el mismo campo. Un mapeo $\mu: E \rightarrow F$ es llamado un morfismo de álgebras si $\mu$ es un mapeo lineal y además la multiplicación es preservada; es decir, se tiene que

$$
\mu(x y)=\mu(x) \mu(y) \quad \forall x, y \in E
$$

(homomorfismo o morfismo son términos también usados). Además, si las álgebras $E$ y $F$ tienen elementos unidad $e_{E}, e_{F}$, respectivamente, siempre asumiremos que un morfismo de álgebras preserva la unidad, en el sentido de que $\mu\left(e_{E}\right)=e_{F}$.

El siguiente resultado, a menudo usado en lo siguiente, es directo.

Lema 3.3.2. Sean $E$ y $F$ álgebras sobre el mismo campo, $\mu: E \rightarrow$ $F$ un morfismo de álgebras y $B$ un subconjunto multiplicativo, convexo, balanceado y absorbente de $F$. Entonces $\mu^{-1}(B)$ también es multiplicativo, convexo,balanceado y absorbente; en otras palabras, la imagen inversa, para un morfismo de álgebras, de un a-barril es nuevemente un a-barril. En particular, si $E$ y $F$ son álgebras topológicas y el morfismo $\mu$ es continuo, entonces la imagen inversa de un $m$-barril de F también es un $m$-barril de $E$.

Por otra parte también tenemos lo siguiente

Proposición 3.3.3. Sean $E$ un álgebra y $\left\{\left(E_{\alpha}, \rho_{\alpha}\right)\right\}_{\alpha \in I}$ una familia de álgebras semi-normadas sobre el mismo campo. Además, sea $\mu_{\alpha}: E \rightarrow E_{\alpha}$, para toda $\alpha \in I$, una familia de morfismos de álgebras. Entonces la topología inicial en $E$, esto es, la topología más robusta para la cual los mapeos $\mu_{\alpha}, \alpha \in I$, son continuos, hace a $E$ un álgebra localmente $m$-convexa. 
Demostración. Consideremos los conjuntos $V$ del álgebra $E$ que son de la forma

$$
V=\bigcap_{i=1}^{n} \mu_{\alpha_{i}}^{-1}\left(U_{\rho_{\alpha_{i}}}\left(\varepsilon_{i}\right)\right)
$$

donde los conjuntos $U_{\rho_{\alpha_{i}}}\left(\varepsilon_{i}\right)$ están variando sobre bases locales de las álgebras semi-normadas $\left(E_{\alpha_{i}}, \rho_{\alpha_{i}}\right)$, con $\varepsilon_{i} \leq 1, \forall 1 \leq i \leq n$, y $n \in \mathbb{N}$. Es claro que los conjuntos anteriores definen $a$-barriles en $E$, por el Lema 3.3.2, y, en efecto, una base de filtro de $E$. En consecuencia, por el Teorema 3.1.11, $E$ es un álgebra localmente $m$-convexa cuya topología coincide con la topología inicial definida en $E$ por los mapeos $\mu_{\alpha}, \alpha \in I$.

Observaciones. 1) Dado que los conjuntos $U_{\alpha_{i}}\left(\varepsilon_{i}\right)$ en (3.13) son $m-$ barriles de las álgebras semi-normadas $\left(E_{\alpha_{i}}, \rho_{\alpha_{i}}\right)$ [ver (3.11) y los comentarios], se concluye, por el Lema 3.3.2, que si el álgebra $E$ es topologizada como arriba, los conjuntos (3.13) define una base local de $E$ que consiste de $m$-barriles.

2) Es claro que el álgebra localmente $m$ - convexa $E$ de la Proposición 3.3.3 es Hausdorff si, y sólo si, para todo $x \in E, x \neq 0$, existe $\alpha \in I$ tal que $\rho_{\alpha}\left(\mu_{\alpha}(x)\right)>0$.

El siguiente corolario es una consecuencia directa de lo anterior.

Corolario 3.3.4. Sea $E$ un álgebra y $\left\{\rho_{\alpha}\right\}_{\alpha \in I}$ una familia de semi-normas submultiplicativas definidas en E. Además, sea $E_{\alpha}=\left(E, \rho_{\alpha}\right), \alpha \in I$, la familia de álgebras semi-normadas. Entonces la topología inicial en $E$, con respecto a los mapeos identidad $i_{\alpha}: E \rightarrow E_{\alpha}, \alpha \in I$, define a $E$ como un álgebra localmente $m$ - convexa. En particular, el álgebra $E$ asi topologizada es Hausdorff si, y sólo si, para toda $x \in E, x \neq 0$, existe $\alpha \in I$ tal que $\rho_{\alpha}(x)>0$ (es decir, si, y sólo si, el respectivo espacio localmente convexo $E_{\alpha}$ es Hausdorff). Además se obtiene una base local de la topología de E, que consiste de $m$-barriles, tomando conjuntos de la forma

$$
V=\bigcap_{i=1}^{n} U_{\rho_{\alpha_{i}}}\left(\varepsilon_{i}\right)=\left\{x \in E: \rho_{\alpha_{i}}(x) \leq \varepsilon, 1 \leq i \leq n\right\},
$$

donde $\varepsilon_{i} \leq 1, n \in \mathbb{N}$.

Así, hablando dentro del contexto previo, decimos que $E$ es un álgebra localmente $m$ - convexa cuya topología está definida por una familia $\Gamma=\left\{\rho_{\alpha}\right\}, \alpha \in$ $I$, de semi-normas submultiplicativas. En este sentido se dice ser la topología localmente $m$ - convexa en $E$ definida por la familia $\Gamma=\left\{\rho_{\alpha}\right\}, \alpha \in I$.

Ahora supongamos que $E$ es un álgebra localmente $m$ - convexa cuya topología es definida por una familia de semi-normas submultiplicativas $\Gamma=$ $\left\{\rho_{\alpha}\right\}$. Decimos que $\Gamma$ es una familia fundamental de la topología de $E$ si la siguiente condición es satisfecha: 
para todo conjunto finito de índices $H \subset I$ existe un índice $\alpha \in I$ y un número real $\varepsilon>0$ tal que

$$
\rho_{\alpha}(x) \geq \varepsilon \rho_{i}(x) \quad \forall i \in H \text { y } \forall x \in E .
$$

La definición de una topología localmente $m$ - convexa sobre un álgebra $E$, por medio de una familia apropiada de semi-normas submultiplicativas, como se describió anteriormente, caracteriza a la clase de álgebras topológicas bajo consideración. Esto es, se obtiene una alternativa de la definición 3.1.7 y del Teorema 3.1.11. Así, la descripción "geométrica" de un álgebra localmente $m$ - convexa dada en la sección 3.1, será complementada por una descripción "analítica". Sin embargo necesitamos el resultado siguiente.

Lema 3.3.5. Sea E un álgebra topológica y sea $U$ un $m$-barril de $E$ el cual es una vecindad del $0 \in E$. Además, sea $P_{U}$ la funcional de Minkowsky asociada a $U$. Entonces se tiene que

$$
U=\left\{x \in E: P_{U}(x) \leq 1\right\}
$$

es decir, $U$ coincide con la respectiva semi-bola cerrada unitaria de su funcional de Minkowsky. En consecuencia, por la Proposición 3.1.5, el interior de $U$ es el conjunto

$$
\stackrel{\circ}{U}=\left\{x \in E: P_{U}(x)<1\right\}
$$

Así, existe una correspondencia uno a uno y sobre, es decir una biyección, entre el conjunto de $m$-barriles del álgebra topológica E, los cuales son vecindades del $0 \in E$, y el conjunto de las semi-normas submultiplicativas continuas definidas en E, dadas por (3.15).

Demostración. Sea $U$ un $m$-barril que es vecindad del cero y sea $P_{U}$ su correspondiente funcional de Minkowsky. Entonces tenemos que

$$
\left\{x \in E: P_{U}(x)<1\right\} \subset U \subset\left\{x \in E: P_{U}(x) \leq 1\right\} .
$$

Por ser $U$ cerrado y por la Proposición 3.1.5, tenemos que

$$
\overline{\left\{x \in E: P_{U}(x)<1\right\}}=\left\{x \in E: P_{U}(x) \leq 1\right\}=U \text {. }
$$

(3.16) se sigue de la Proposición 3.1.5 y lo último se sigue por lo anterior.

Así, ahora tenemos la siguiente

Proposición 3.3.6. Sea E un álgebra localmente $m$ - convexa (definición 3.1.4 y/o Teorema 3.1.11). Entonces existe una familia fundamental (de seminormas submultiplicativas) de la topología de E. 
Demostración. Dado que $E$ es un álgebra localmente $m$ - convexa entonces, por el Teorema 3.1.11, $E$ es un álgebra topológica con una base local que consiste de $m$-barriles. Sea $\beta=\{U\}$ tal base local. Como $0 \in U$, $\forall U \in \beta$ entonces, por el Lema 3.3.5, las funcionales de Minkowsky $P_{U}$ constituyen una familia de semi-normas submultiplicativas continuas, donde $U=$ $\left\{x \in E: P_{U}(x) \leq 1\right\}$. Sea $\Gamma=\left\{P_{U}\right\}, U \in \beta$. En consecuencia, por el Corolario 3.3.4, la familia $\Gamma$ define la misma topología localmente $m$ - convexa sobre $E$ que la familia $\beta$. Por otra parte, por (3.14) y por Lema 3.2 (ver [Ma; pag. 15]), se concluye que $\Gamma$ es una familia fundamental de la topología de $E$.

Como mencionamos antes, el siguiente resultado básico combina las dos definiciones precedentes de un álgebra localmente $m$ - convexa; a saber, la "geométrica" y la "analítica".

Teorema 3.3.7. Sea E un álgebra. Entonces, las siguientes afirmaciones son equivalentes:

1) E es un álgebra localmente $m$ - convexa (definición 3.1.7)

2) E es un álgebra topológica con una base local que consiste de $m$-barriles

3) Existe una familia fundamental de semi-normas submultiplicativas en E.

Demostración. Por Teorema 3.1.11, (1) es equivalente a (2). Por Proposición 3.3.6, Corolario 3.3.4 y por Lema 3.2 (ver [Ma; pag. 15]), (2) es equivalente a (3) .

Así, sobre la base del resultado anterior, para examinar si un álgebra dada, siendo un espacio vectorial topológico, es localmente $m$ - convexa, se aplica indistintamente las afirmaciones (2) o (3) del teorema anterior, de acuerdo a como lo sugiera el caso particular a tratar.

\subsection{Ciertas clases particulares de álgebras topo- lógicas}

En esta sección consideraremos álgebras de Waelbroeck, $Q$-álgebras y otros tipos más de álgebras topológicas que usaremos en el capítulo siguiente. Discutiremos aquí sus propiedades más básicas y algunos resultados sólo se mencionarán sin ser demostrados. Para conocer más a fondo de el tipo de álgebras que vamos a considerar, ver [Ma].

Definición 3.4.1. Un álgebra topológica $E$ con unidad se dice ser una $Q$-algebra si el conjunto de los elementos invertibles de $E$, denotado por $G(E)$, es un subconjunto abierto de E. 
Observación. La definición anterior es usualmente aplicada, en forma más extensa, a álgebras topológicas que no necesariamente tienen elemento unidad, empleando el concepto de "cuasi-regularidad" de elementos de un álgebra.

Lema 3.4.2. Sea $E$ un álgebra topológica y sea $G(E)$ el conjunto de los elementos invertibles de E. Entonces las siguientes afirmaciones son equivalentes

1) $G(E)$ es un subconjunto abierto de $E$; es decir, E es una $Q$-álgebra

2) $G(E)$ tiene interior no vacío

3) $G(E)$ es vecindad de $e \in E$.

Demostración. Es claro que (1) implica (2), mientras que como $e=e \cdot e$ entonces $e$ es un elemento de $G(E)$ que, por ser abierto, es vecindad de $e$. Así (1) implica (3). Es obvio que (3) implica (2). Sólo resta probar que (2) implica (1). Esto es también inmediato.

Nuestra tarea en lo que sigue es examinar qué propiedades fundamentales, las cuales son válidas para las álgebras de Banach, aun son verdaderas para álgebras topológicas. Comenzamos con la siguiente

Definición 3.4.3. Dada un álgebra topológica $E$ con unidad, diremos que $E$ tiene una inversa continua si el mapeo ${ }^{-1}: G(E) \rightarrow G(E), x \rightarrow x^{-1}$, es continuo.

$\mathrm{Al}$ respecto tenemos el siguiente

Lema 3.4.4. Sea $E$ un álgebra localmente $m$ - convexa con elemento unidad. Entonces $E$ tiene una inversa continua.

Demostración. Por ser $E$ un álgebra $m$ - convexa entonces, por Teorema 3.3.7 (3), existe una familia fundamental de semi-normas submultiplicativas sobre $E$. Sea $\Gamma=\left\{\rho_{\alpha}\right\}, \alpha \in I$, tal familia e $I$ un conjunto de índices. Sea $x \in G(E), x+h \in G(E)$, considerese $(x+h)^{-1}=x^{-1}+k$ y sea $\alpha \in I$ arbitraria. Demostremos que si $\rho_{\alpha}(h)$ es pequeño, entonces $\rho_{\alpha}(k)$ es pequeño. Dado que

$$
e=\left(x^{-1}+k\right)(x+h)=x^{-1} x+x^{-1} h+k x+k h=e+x^{-1} h+k h+k x,
$$

de donde

$$
x^{-1} h+k h+k x=0 .
$$

Multiplicando la ecuación anterior por la derecha por $x^{-1}$ tenemos que $x^{-1} h x^{-1}+k h x^{-1}+k x x^{-1}=0$, de donde se tiene que $k=-x^{-1} h x^{-1}-k h x^{-1}$. Esto implica que

$$
\rho_{\alpha}(k) \leq \rho_{\alpha}\left(x^{-1} h x^{-1}\right)+\rho_{\alpha}\left(k h x^{-1}\right) \leq \rho_{\alpha}\left(x^{-1}\right)^{2} \rho_{\alpha}(h)+\rho_{\alpha}\left(x^{-1}\right) \rho_{\alpha}(h) \rho_{\alpha}(k) .
$$


Si $\rho_{\alpha}\left(x^{-1}\right) \rho_{\alpha}(h)<1$, entonces tenemos que

$$
\rho_{\alpha}(k) \leq \frac{\rho_{\alpha}\left(x^{-1}\right)^{2}}{1-\rho_{\alpha}(h) \rho_{\alpha}\left(x^{-1}\right)} \rho_{\alpha}(h) .
$$

Para $0<\varepsilon<\rho_{\alpha}\left(x^{-1}\right)$, tomemos $\delta=\left[2 \rho_{\alpha}\left(x^{-1}\right)^{2}\right]^{-1} \varepsilon$. Entonces, para $\rho_{\alpha}(h)<\delta$ y $\rho_{\alpha}\left(x^{-1}\right) \rho_{\alpha}(h)<\delta \rho_{\alpha}\left(x^{-1}\right)<\frac{1}{2}$, tenemos que

$$
\rho_{\alpha}(k)<2 \rho_{\alpha}\left(x^{-1}\right)^{2} \rho_{\alpha}(h)<2 \rho_{\alpha}\left(x^{-1}\right)^{2} \delta=\varepsilon .
$$

La operación inversa, en un álgebra topológica con unidad, puede ser continua sin que el álgebra sea localmente $m$ - convexa, como esto es demostrado en el siguiente resultado. De hecho, el mismo resultado puede ser considerado como un ejemplo más concreto de una situación más general (ver la observación que se menciona abajo).

Teorema 3.4.5. Sea $E$ un álgebra topológica metrizable con unidad para la cual el grupo $G(E)$ de los elementos invertibles es, en la topología relativa, un espacio de Baire separable. Entonces el mapeo inversión en $E$ es continuo sobre $G(E)$ (E-valuado), asi que $G(E)$ es, en particular, un grupo topológico con respecto a la topología relativa.

Demostración. Dado que $E$ es un álgebra topológica metrizable y $G(E)$ es un espacio de Baire separable, en la topología relativa, entonces $G(E)$ es un espacio de Baire que es también metrizable y separable. En consecuencia $G(E)$ es segundo numerable. Además, por ser $G(E)$ metrizable, este también es un espacio $T_{4}$ (es decir, un espacio topológico normal y todo punto, considerado como un conjunto, es cerrado). Esto implica que $G(E)$ es $T_{3}$ (es decir, un espacio topológico regular y todo punto, considerado como un conjunto, es cerrado). En consecuencia $G(E)$ es un grupo semitopológico regular que satisface el segundo axioma de numerabilidad. Esto implica que $G(E)$ es de tipo $C$ (ver [Hu;Teorema 9, pag. 37]). Por lo tanto $G(E)$ es un grupo topológico (ver [Hu;Teorema 8, pag. 36]). Así, la operación inversa es continua.

Como una aplicación se obtiene el siguiente

Corolario 3.4.6. Sea $E$ un álgebra de Frechet con elemento unidad para la cual el grupo $G(E)$ de elementos invertibles es un $G_{\delta}$ - conjunto separable. Entonces $E$ tiene una inversa continua, asi que $G(E)$ es, en particular, un grupo topológico en la topología relativa.

Demostración. Dado que $E$ es un álgebra de Fréchet entonces, en particular, es un espacio vectorial topológico de Fréchet, y como $G(E)$ es un $G_{\delta}$ - conjunto esto implica que $G(E)$ es un subespacio metrico completo (ver 
[Tk;pag. 102]). En consecuencia, $G(E)$ es un espacio de Baire. Así, $G(E)$ es un espacio de Baire separable. Por el Teorema 3.4.5, $G(E)$ es un grupo topológico.

Por otra parte, también obtenemos el siguiente

Corolario 3.4.7. Sea E una Q-álgebra de Baire metrizable con unidad. tal que $G(E)$ sea un subespacio separable. Entonces $G(E)$ es, en la topología relativa, un grupo topológico. Así que E tiene, además, una inversa continua.

En particular una $Q$-álgebra de Fréchet separable con unidad es un álgebra topológica con una inversa continua dando al grupo de los elementos invertibles una estructura de grupo topológico en la topología relativa.

Demostración. Dado que $G(E)$ es abierto y $E$ es, en particular, un espacio vectorial topológico de Baire, entonces $G(E)$ es un espacio de Baire (ver [GT;pag. 115]). Además, por ser $G(E)$ separable y por Teorema 3.4.5, $G(E)$ es un grupo topológico en la topología relativa, de donde $E$ tiene una inversa continua.

Para la segunda parte, por ser, en particular, $E$ un espacio vectorial topológico de Fréchet, entonces se tiene que $E$ es un espacio de Baire,y por ser $G(E)$ abierto, entonces $G(E)$ es también un espacio de Baire. Por ser $E$ separable entonces $G(E)$ es, en la topología relativa, también separable. Por el Teorema 3.4.5, $G(E)$ es un grupo topológico.

Observación. De acuerdo al resultado de T. Husain, aplicado en la demostración del Teorema 3.4.5, un grupo $G$, dotado con la topología $\tau$ que hace a la "multiplicación en $G$ " (la operación de grupo) separadamente continua (es decir, un grupo semitopológico) se convierte en un grupo topológico si $G$ es un espacio de Baire normal segundo numerable en la topología $\tau$. Además, por un resultado conocido de D. Montgomery [Mo;pag. 881, Teorema 2], un grupo semitopológico cuyo espacio topológico base es metrizable completo y separable, es un grupo topológico. Ahora, esta condición es satisfecha si $E$ es un álgebra de Fréchet con unidad y $G(E)$, el grupo de los elementos invertibles de $E$, es un $G_{\delta}-$ conjunto separable.

Más generalmente, la última conclusión es válida si $E$ es un álgebra topológica metrizable con elemento unidad y $G(E)$ es localmente completo y separable. A este respecto notaremos, por [Hu;pag. 36, Teorema 7], que uno concluye lo siguiente:

Toda álgebra topológica $E$ con unidad en la cual el grupo $G(E)$ es un espacio Baire segundo numerable tiene una inversa continua, y $G(E)$ es, en consecuencia, un grupo topológico en la topología relativa.

En particular, la última conclusión es válida para una $Q$-álgebra de Baire con unidad y $G(E)$ segundo numerable o, más particularmente, si $E$ es una $Q$-álgebra de Baire metrizable separable con unidad. 
Las álgebras topológicas que exhiben las propiedades de las álgebras que aparecen en la segunda parte del Corolario 3.4.7 jugarán un papel importante en lo siguiente y en el capítulo 4. En consecuencia, definimos a tales álgebras. Estas álgebras han sido aplicadas (en particular como localmente convexas y/o localmente $m$-convexas) en los inicios de la década de los cincuenta por L. Waelbroeck.

Por otra parte, las mismas álgebras, al menos las localmente $m$-convexas, parecen constituir esa clase particular de álgebras topológicas en las cuales se pueden encontrar muchos de los resultados fundamentales de la teoría clásica de las álgebras de Banach. La misma clase de álgebras juega un papel significante en otras partes de la teoría de álgebras topológicas o de sus aplicaciones. Comenzamos dando la definición formal.

Definición 3.4.8. Por un álgebra de Waelbroeck queremos decir un álgebra topológica $E$ con un elemento unidad e de tal manera que el grupo $G(E)$ de los elementos invertibles en $E$ es un conjunto abierto en $E$ y el mapeo inverso es continuo sobre $G(E)$.

En otras palabras, un álgebra de Waelbroeck es una $Q$-álgebra con inversa continua. Además, por lo precedente (ver la última observación) una $Q$-álgebra de Baire separable (en consecuencia, en particular, una $Q$-álgebra de Fréchet separable) con unidad es un álgebra de Waelbroeck.

Podemos formular otra versión útil de la definición 3.4.8 vía la siguiente

Proposición 3.4.9. Sea E un álgebra topológica con unidad e. Entonces los siguientes enunciados son equivalentes:

1) E es un álgebra de Waelbroeck

2) El grupo $G(E)$ de elementos invertibles de $E$ es una vecindad de e y el mapeo inverso en $E$ es continuo en e

3) El elemento unidad e tiene una vecindad que consiste de elementos invertibles y el mapeo inverso en $E$ es continuo en e.

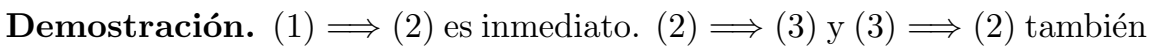
son inmediatas. Sólo tenemos que demostrar $(2) \Longrightarrow(1)$. Por el Lema 3.4.2 y por (2) $E$ es una $Q$-álgebra. Por lo tanto es suficiente probar que $E$ tiene una inversa continua, siendo ésta continua en $e$. Sea $x_{0} \in G(E), x_{0} \neq e$, veamos que el mapeo inverso es continuo en $x_{0}$ si, y sólo si, $\forall U$ vecindad de $x_{0}^{-1} \exists V$ vecindad de $x_{0}$ tal que $V^{-1} \subset U$.

Como $x_{0} \in G(E)$, sea $U$ vecindad de $x_{0}^{-1}$ contenida en $G(E)$ (esto es válido por ser $G(E)$ abierto). Dado que $x_{0} \neq e$, tomemos la siguiente regla de correspondencia: $x \rightarrow e+x_{0}^{-1}\left(x-x_{0}\right), x \in E$.

Como $E$ es un álgebra topológica, entonces el mapeo anterior es continuo en $x=x_{0}$, asi que existe $W$ vecindad del cero de $E$ tal que $e+x_{0}^{-1}\left(x-x_{0}\right) \in G(E)$, 
$\forall x-x_{0} \in W$; es decir $e+x_{0}^{-1}\left(x-x_{0}\right) \in G(E), \forall x \in x_{0}+W$, donde $x_{0}+W$ es vecindad de $x_{0}$ en $E$. Así, debido a que la relación

$$
x=x_{0}\left[e+x_{0}^{-1}\left(x-x_{0}\right)\right]
$$

es válida para toda $x \in E$, el mapeo

$$
x \rightarrow\left[e+x_{0}^{-1}\left(x-x_{0}\right)\right]^{-1},
$$

definido para toda $x \in x_{0}+W$, es, por hipótesis, continuo en $x=x_{0}$. En consecuencia, tenemos que

$$
x^{-1}=\left[e+x_{0}^{-1}\left(x-x_{0}\right)\right]^{-1} x_{0}^{-1},
$$

con $x \in x_{0}+U$. En consecuencia tenemos la continuidad deseada del mapeo $x \rightarrow x^{-1}, x \in E$, en el punto $x=x_{0}$.

Sobre la base de la demostración previa, se concluye que la condición (3) de la Proposición 3.4.9 puede ser enunciada para todo elemento invertible $x_{0} \in E$, la cual es, en efecto, una forma equivalente de la condición (1) de la misma proposición; es decir, de la definición 3.4.8.

Ahora, como una aplicación de lo anterior, estamos en posición de enunciar el siguiente

Teorema 3.4.10. Sean E un álgebra de Waelbroeck y $x \in E$ arbitrario. Entonces el conjunto resolvente $p(x)$ del elemento $x$ es un subconjunto abierto de $\mathbb{C}$ y la respectiva función resolvente $R(x ; \cdot)$ es un mapeo holomorfo E-valuado sobre $p(x)$, la cual también satisface la relación

$$
\lim _{\lambda \rightarrow \infty} R(x ; \lambda)=0
$$

("acotado en el punto al infinito" de $\mathbb{C}$ ), asi que éste es, en particular, un mapeo (holomorfo) acotado sobre $p(x)$.

Demostración. Por ser $E$ álgebra de Waelbroeck y por definición de

$$
R(x ; \cdot): p(x) \subset \mathbb{C} \rightarrow E, \lambda \rightarrow(\lambda-x)^{-1},
$$

claramente $R(x ; \cdot)$ es una función continua. Además, por ser $G(E)$ abierto y $[R(x ; \cdot)]^{-1}[G(E)]=p(x)$, entonces $p(x)$ es abierto en $\mathbb{C}$. Veamos que $R(x ; \cdot)$ es un mapeo holomorfo. Sean $\lambda_{0}, \lambda \in p(x), \lambda \neq \lambda_{0}$, por probar que

$$
\lim _{\lambda \rightarrow \lambda_{0}} \frac{R(x ; \lambda)-R\left(x ; \lambda_{0}\right)}{\lambda-\lambda_{0}}
$$

existe. Usando la definición de la función resolvente se prueba que lo siguiente igualdad se cumple

$$
R(x ; \lambda)-R\left(x ; \lambda_{0}\right)=-\left(\lambda-\lambda_{0}\right) R(x ; \lambda) R\left(x ; \lambda_{0}\right),
$$


de donde, haciendo $\lambda \rightarrow \lambda_{0}$ y por ser $R(x ; \cdot)$ continua en $p(x)$, tenemos que

$$
\lim _{\lambda \rightarrow \lambda_{0}} \frac{R(x ; \lambda)-R\left(x ; \lambda_{0}\right)}{\lambda-\lambda_{0}}=-\lim _{\lambda \rightarrow \lambda_{0}} R(x ; \lambda) R\left(x ; \lambda_{0}\right)=-\left[R\left(x ; \lambda_{0}\right)\right]^{2} .
$$

Así, $R(x ; \cdot)$ es un mapeo diferenciable con valores en $E$ definido sobre $p(x)$. En consecuencia, por definición, es un mapeo holomorfo sobre $p(x)$. Dado que $E$, en particular, es un espacio vectorial topológico entonces el mapeo $\alpha \rightarrow e-\alpha x$ es continuo de $\mathbb{C}$ en $E$. Por lo tanto existe una vecindad $N_{\varepsilon}(0)$ del cero de $\mathbb{C}$ tal que $e-\alpha x \in G(E), \forall \alpha \in N_{\varepsilon}(0)$. En consecuencia la relación

$$
\begin{aligned}
R(x ; \lambda) & =(\lambda-x)^{-1}=\left\{\lambda\left(e-\lambda^{-1} x\right)\right\}^{-1}=\lambda^{-1}\left(e-\lambda^{-1} x\right)^{-1} \\
& =\frac{1}{\lambda}\left(e-\frac{1}{\lambda} x\right)^{-1}
\end{aligned}
$$

tiene sentido para $\alpha=\frac{1}{\lambda},|\alpha|=\left|\frac{1}{\lambda}\right|<\varepsilon$; es decir $|\lambda|>\varepsilon$. Así, tomando el límite en la última relación para $\lambda \rightarrow \infty$ en $\mathbb{C}$, tenemos que

$$
\lim _{\lambda \rightarrow \infty} R(x ; \lambda)=(\lambda-x)^{-1}=\lim _{\lambda \rightarrow \infty} \frac{1}{\lambda}\left(e-\frac{1}{\lambda} x\right)^{-1}=0 .
$$

Observación. En la prueba previa un mapeo con valores en $E$ y definido sobre un subconjunto abierto de $\mathbb{C}$ fue tomado como "holomorfo" siendo diferenciable sobre su dominio de definición (y en consecuencia también continua; este fue el caso para el mapeo $R(x ; \cdot))$. Esto recuerda, por cierto, el caso $E=\mathbb{C}$, a saber, el de los mapeos holomorfos de valor complejo, mientras que esto aun es válido si se considera la composición de un mapeo holomorfo con valores en $E$ con una forma lineal continua (de valor complejo) sobre el álgebra topológica $E$; es decir, con elementos del dual topológico $E^{\prime}$ de $E$. Al respecto, sería de importancia tener en $E$ un "buen abastecedor" de tales formas, como esto es, por ejemplo, si $E$ es un álgebra localmente convexa (Hahn-Banach).

Pero esto aún puede suceder en álgebras topológicas las cuales no necesariamente son localmente convexas. Así, lo que realmente se necesita en este contexto es un álgebra topológica $E$ que admita un conjunto total de formas lineales continuas, en el sentido de que exista un subconjunto $A$ de $E^{\prime}$, tal que para todo $x \in E, x \neq 0$, exista un elemento $f \in A$ con $f(x) \neq 0$.

Así, como ya se notó, si $E$ es, en particular, un álgebra localmente convexa entonces $E^{\prime}$ es un conjunto total para $E$ (Hahn-Banach): de hecho, esto es válido para todo subconjunto $A$ de $E^{\prime}$ cuyo casco lineal es "debilmente denso" en $E^{\prime}$; es decir, si $A$ es un conjunto total en $E_{s}^{\prime}$, el dual topológico débil de $E$.

Finalmente, en conexión con el Teorema 3.4.10, aun notamos que por la prueba del mismo teorema, existe una vecindad del punto al infinito de $\mathbb{C}$ contenida en $p(x)$; así que $p(x)$ es una vecindad del punto al infinito de $\mathbb{C}$. Además, 
se puede probar que $R(x ; \cdot)$ es holomorfa también en ese punto (ver [Na; pag. 172, cap. III y pag. 66]).

Como una aplicación de la discusión anterior, llegamos al siguiente resultado básico el cual es válido, de hecho, para una clase apropiada de álgebras topológicas (para las localmente convexas, Corolario 3.4.12) que tiene una inversa continua. Esto es, tenemos el

Teorema 3.4.11. Sea E un álgebra topológica con unidad y con inversa continua que admite, además, un conjunto total de formas lineales continuas (ver la observación previa). Entonces se tiene que

$$
S p_{E}(x) \neq \emptyset,
$$

para todo $x \in E$.

Demostración. Supongamos que la conclusión es falsa, entonces $S p_{E}(x)=$ $\emptyset$, para algún $x \in E$. Como el conjunto resolvente de $x$ es $p(x)=\mathbb{C} \backslash S p_{E}(x)$, entonces $p(x)=\mathbb{C}$, asíque la función resolvente $R(x ; \lambda)$ está definida en todo $\mathbb{C}$. Además, $R(x ; \lambda)$ es holomorfo y acotado. En consecuencia, si $A \subset E^{\prime}$ es un conjunto total de formas lineales continuas, entonces el mapeo $\lambda \rightarrow f(R(x ; \lambda))$, con $f \in A$, es un mapeo holomorfo de valor complejo definido sobre $\mathbb{C}$ ( es decir, una función entera) la cual es también acotada, puesto que $f$ es continua y $R(x ; \lambda)(\mathbb{C})$ es acotado en $E$, lo cual implica que $(f(R(x ; \cdot)))(\mathbb{C})$ es acotado. Por el Teorema de Louville, el mapeo $\lambda \rightarrow f(R(x ; \lambda))$ es constante. Pero por (3.17) tenemos que

$$
f(R(x ; \lambda))=f\left((\lambda-x)^{-1}\right)=0,
$$

para todo $f \in A$ y $\lambda \in \mathbb{C}$. Así, por hipótesis para $A$ y por lo anterior, $(\lambda-x)^{-1}=0$. Esto es una contradicción $\mathrm{y}$, por lo tanto, el teorema queda demostrado.

El siguiente resultado fundamental es una consecuencia directa del resultado anterior y del Lema 3.4.4

Corolario 3.4.12. Sea $E$ un álgebra localmente convexa con unidad y con inversa continua. Entonces el espectro $S p_{E}(x)$ de cualquier elemento $x \in E$ es un subconjunto no vacío de $\mathbb{C}$. En particular, si $E$ es un álgebra localmente $m$ - convexa con unidad, todo elemento de E tiene espectro no vacío.

El siguiente resultado nos dice bajo qué condiciones sobre $E$ todo elemento de $E$ tiene espectro compacto (posiblemente vacío).

Proposición 3.4.13. Sea E una Q-álgebra. Entonces, para todo elemento $x \in E$, el espectro de $x$ (posiblemente vacio) es un subconjunto compacto de $\mathbb{C}$. Por otra parte, para cualquier álgebra de Waelbroeck localmente convexa 
tenemos que, en particular para $Q$-álgebras localmente $m$-convexas (ver Lema 3.4.4), el espectro de cualquier elemento $x \in E$ es un subconjunto compacto no vacío de $\mathbb{C}$.

Demostración. Ver [Ma; pag. 60, Proposición 4.2].

Consideremos ideales maximales en álgebras topológicas. Sabemos que todos los ideales maximales en álgebras de Banach son cerrados. El siguiente resultado afirma que ese resultado también es cierto para $Q$-álgebras. Esto es, tenemos el

Teorema 3.4.14. Sea E una Q-álgebra con unidad. Entonces todo ideal I maximal de E es cerrado.

Demostración. Sea $I$ ideal maximal de $E$. Por ser $I$ ideal y por Corolario 3.1.10, tenemos que $\bar{I}$ es ideal de $E$. Dado que $G(E)$ es abierto, entonces $I \cap$ $G(E)=\emptyset$. De donde $I \subset E \backslash G(E)$. Así $\bar{I} \subset E \backslash G(E)$. Por lo tanto $\bar{I} \cap G(E)=\emptyset$ y $I \subset \bar{I}$, lo cual implica que $I=\bar{I}$.

La situación descrita por el teorema anterior puede ser diferente para un álgebra topológica arbitraria (igual para un álgebra localmente $m$-convexa) la cual no es una $Q$-álgebra; es decir, pueden existir ideales maximales los cuales son subconjuntos densos del álgebra topológica dada.

Lema 3.4.15. Sea E un álgebra topológica con unidad con inversa continua e I un ideal bilateral cerrado de E. Entonces el álgebra cociente E/I es un álgebra topológica con inversa continua.

Demostración. Ver [Ma; pag. 71] 


\section{Capítulo 4}

\section{Fórmula del mapeo espectral para álgebras de Waelbroeck y sus subálgebras}

Las álgebras de Waelbroeck localmente convexas son muy cercanas a las álgebras de Banach, en el sentido de la teoría espectral. Todo elemento de un álgebra de este tipo tiene un espectro compacto no trivial. Además, el espectro combinado, definido exáctamente como el espectro de Harte, tiene sentido para álgebras de Waelbroeck localmente convexas y satisface la fórmula del mapeo espectral.

En el presente capítulo consideramos clases de espectros combinados sobre subálgebras de un álgebra de Waelbroeck localmente convexa, dando la fórmula del mapeo espectral para ellos.

El tema de sección 4.1 es el estudio de la propiedad de la proyección de una familia $I_{E}^{l}(A)$ de ideales izquierdos en una subálgebra $A \subset E$ que consisten de elementos no invertibles en $E$.

Para $E$ un álgebra de Waelbroeck localmente convexa con unidad y para $A$ tal que $a A a^{-1} \subset A$, para todo $a \in A \cap G(E)$, donde $G(E)$ es el conjunto de los elementos invertibles en $E$, demostramos que $I_{E}^{l}(A)$ tiene la propiedad de la proyección y, en consecuencia usando el Teorema 2.1.1, el correspondiente espectro $S p_{I_{E}^{l}(A)}$ satisface la fórmula del mapeo espectral. 


\subsection{Los ideales de $A \subset E$ que consisten de ele- mentos no invertibles en $E$}

Primero estudiamos la propiedad de la proyección de la familia $I_{E}^{l}(A)$, para $E$ una $Q$-álgebra con unidad y $A \subset E$ una subálgebra con unidad tal que $a A a^{-1} \subset A$, para toda $a \in A \cap G(E)$. De esto se sigue que $I_{E}^{l}(A)$ tiene la propiedad de la proyección si la familia de todos los ideales izquierdos en $R(A)$ tiene la propiedad de la proyección.

Teorema 4.1.1. Sean $E$ una $Q$-álgebra con unidad y $A \subset E$ su subálgebra con unidad. Supongamos que $a A a^{-1} \subset A$, para toda $a \in A \cap G(E)$. Entonces

(1) La subálgebra $R(A)$ es cerrada con respecto a la operación inverso en $E$.

(2) Si $I \in I_{E}^{l}(A)$ y $J$ es el ideal izquierdo cerrado generado por $I$ en $R(A)$, entonces $J \in I_{E}^{l}(R(A))$. En particular $J$ es un ideal propio.

Demostración. Sea

$$
\widetilde{E}=\left\{b^{-1} a: a \in A, b \in A \cap G(E)\right\} .
$$

Obviamente $\widetilde{E} \subset R(A), A \subset \widetilde{E}$ y $\left\{b^{-1}: b \in A \cap G(E)\right\} \subset \widetilde{E}$. Un elemento $x=b^{-1} a \in \widetilde{E}$ es invertible en $E$ si, y sólo si, $a$ es invertible en $E$ y este es el caso $x^{-1} \in E$. El espacio $\widetilde{E}$ es cerrado bajo la operación inverso en $E$. Gracias a la propiedad $a A a^{-1} \subset A$, para $a \in A \cap G(E)$, se sigue que el espacio $\widetilde{E}$ es cerrado con respecto a la multiplicación:

$$
r^{-1} a s^{-1} b=r^{-1} s^{-1} s a s^{-1} b=(s r)^{-1} s a s^{-1} b \in \widetilde{E},
$$

por que $\operatorname{sas}^{-1} b \in A$.

$\widetilde{E}$ es también un espacio vectorial por que

$$
r^{-1} a+s^{-1} b=(s r)^{-1}\left(s a+(s r) r(s r)^{-1} b \in \widetilde{E},\right.
$$

dado que $s a+(s r) r(s r)^{-1} b \in A$.

De esto se sigue que $R(A)$ es igual a $\widetilde{E}$ en $E$; asi que es también cerrado bajo la operación inverso en el álgebra $E$. El ideal $J$ generado en $R(A)$ por $I \in I_{E}^{l}(A)$ es la cerradura del ideal $J_{0}=\left\{s^{-1} a: a \in I, s \in A \cap G(E)\right\}$. Los elementos de $J_{0}$ no son invertibles en $E$. Lo mismo es cierto para $J$ por que $E$ es una $Q$-álgebra.

Teorema 4.1.2. Sea E una $Q$-álgebra con unidad y $A$ una subálgebra con unidad de $E$ tal que aAa ${ }^{-1} \subset A$, para $a \in A \cap G(E)$. Si $I^{l}(R(A))$ tiene la propiedad de la proyección, entonces la familia $I_{E}^{l}(A)$ tiene la propiedad de la proyección. 
Demostración. Supongamos lo contrario. Entonces existen $a_{1}, \ldots, a_{k} \in I \in$ $I_{E}^{l}(A)$ y $c \in A$ tales, que para todo $\lambda \in \mathbb{C}$, un elemento de la forma

$$
u=\sum_{j=1}^{k} x_{j} a_{j}+x_{k+1}(c-\theta e), \quad x_{j} \in A, 1 \leq j \leq k+1,
$$

es invertible en $E$, asi que también lo es en $R(A)$. Para toda $\lambda$ el ideal generado en $R(A)$ por $a_{1}, \ldots, a_{k}, c-\lambda e$ es $R(A)$. Sin embargo, por el Teorema 4.1.1, el ideal generado en $R(A)$ por $a_{1}, \ldots, a_{k}$ es propio. En consecuencia $I^{l}(R(A))$ no tiene la propiedad de la proyección. La contradicción prueba el teorema.

Observemos que no se supone que $A$ sea cerrada en $E$. Obviamente la versión con ideales derechos propios también es cierta.

La propiedad de la proyección de la familia de todos los ideales izquierdos para un álgebra de Waelbroeck localmente convexa fue estudiada en [W3].

Teorema 4.1.3. Si E es un álgebra de Waelbroeck localmente convexa, entonces $I^{l}(E)$ tiene la propiedad de la proyección.

Corolario 4.1.4. Sea E un algebra de Waelbroeck localmente convexa con unidad y sea $A$ una subálgebra de $E$ con unidad tal que $a A a^{-1} \subset A$, para toda $a \in A \cap G(E)$. Entonces $I_{E}^{l}(A)$ tiene la propiedad de la proyección. El correspondiente espectro combinado $S p_{I_{E}^{l}(A)}$ satisface la fórmula del mapeo espectral.

Demostración. Una subálgebra, cerrada bajo la operación inverso, de un álgebra de Waelbroeck es un álgebra de Waelbroeck, asi que $R(A)$ es un álgebra de Waelbroeck localmente convexa. Por el Teorema 4.1.3, la clase $I^{l}(R(A))$ tiene la propiedad de la proyección, en consecuencia $I_{E}^{l}(A)$ tiene la propiedad de la proyección por el Teorema 4.1.2. La fórmula del mapeo espectral para el espectro $S p_{I_{E}^{l}(A)}$ se sigue por el Teorema 2.1.1.

\subsection{Casos especiales y aplicaciones}

Sea $E$ un álgebra de Waelbroeck localmente convexa con unidad y $A$ su subálgebra conmutativa con unidad. Si $a \in A \cap G(E)$ entonces $a^{-1}$ conmuta con todos los elementos de $A$ y la condición $a A a^{-1} \subset A$ es satisfecha.

Teorema 4.2.1. Sea A una subálgebra conmutativa con unidad de un álgebra de Waelbroeck E. Entonces para cualquier $I \in I_{E}(A)$ existe $J \in I_{E}(A)$ el cual es de codimensión uno en A y contiene a $I$. 
La prueba se sigue por los mismos argumentos que son usados en el Teorema 2.4.1 para el caso de álgebras de Banach, asi que se omite la misma. La herramienta principal, la cual también es válida en el caso de álgebras de Waelbroeck, es esa para elementos que conmutan mutuamente $a_{1}, \ldots, a_{k} \in I$ y para $c \in A$ que conmuta con todos los $a_{i}$, el conjunto

$$
\partial\left(a_{1}, \ldots, a_{k}\right)=\left\{\lambda \in \mathbb{C}: I_{A}\left(a_{1}, \ldots, a_{k}, c-\lambda e\right) \in I_{E}(A)\right\}
$$

es no vacío y compacto. Aquí $I_{A}\left(a_{1}, \ldots, a_{k}, c-\lambda\right)$ denota el ideal generado en $A$ por los correspondientes elementos.

Recordemos una aplicación típica del Teorema 4.2.1 en el caso de un álgebra de Banach conmutativa.

Teorema 4.2.2. $[W 1]$. Sea $A$ un álgebra de Banach conmutativa con unidad. Todo ideal que consiste de divisores topológicos de cero está contenido en un ideal maximal de A que también consiste de divisores topológicos de cero.

Demostración. Denotemos por $M(A)$ al espacio de ideales maximales de $A$ dotado con la topología de Gelfand y por^: $A \rightarrow C(M(A))$, dada por $a \rightarrow \widehat{a}$, la transformada de Gelfand. Denotemos por $g$ al homomorfismo que asocia a $a \in A$ a la restricción de $\widehat{a}$ a la corteza cor $A$. Recordemos que cor $A$ es el subconjunto compacto de $M(A)$ que contiene a todos los ideales maximales que consisten de DTC combinados de $A$. Cualquier divisor topológico de cero está contenido en algún $J \in \operatorname{cor} A$. Sea $A_{1}=g(A)$ y sea $B=C(\operatorname{cor} A)$ el espacio de todas las funciones continuas definidas en $\operatorname{cor} A$.

Un ideal $I$ de $A$ consiste de divisores topológicos de cero si, y sólo si, $g(I)$ consiste de elementos no invertibles en el álgebra de Banach $B$. Por el Teorema 4.2.1, $I$ está contenido en un ideal $J$ de codimensión uno en $A_{1}$ que consiste de elementos los cuales alcanzan el cero en cor $A$. La imagen inversa $g^{-1}(J)$ es un ideal maximal en $A$ que contiene a $I$ y consiste de divisores topológicos de cero de $A$.

Regresando al caso no conmutativo, describimos una construcción simple la cual conduce a que el par $A \subset E$ satisfaga la condición $(C): a A a^{-1} \subset A$ para todo $a \in A \cap G(E)$.

Proposición 4.2.3 Consideremos el par de álgebras con unidad $A \subset B$ que satisfagan la propiedad $(C)$. Si $D$ es un álgebra con unidad y $g: D \rightarrow B$ es un homomorfismo suprayectivo, entonces $\widetilde{A}=g^{-1}(A) \subset D$ es un par que satisface la condición $(C)$.

Demostración. Si $a \in \widetilde{A}$ y $b \in G(D)$, entonces $g\left(b a b^{-1}\right)=g(b) g(a) g(b)^{-1}$ $\in A$, por suposición. En consecuencia $b a b^{-1} \in \widetilde{A}$. 


\section{Capítulo 5}

\section{La propiedad de la proyección de una familia de ideales y la fórmula del mapeo espectral en álgebras localmente m-convexas}

En el presente capítulo consideramos una clase de espectro combinado sobre álgebras localmente $m$ - convexas complejas con unidad, definido para una familia de ideales izquierdos apropiados sobre tales álgebras, demostrando la fórmula del mapeo espectral.

Teorema 5.1.1. Sea $E$ un álgebra $m$ - convexa compleja con unidad. Supongamos que para toda $(k+m)$ - upla de elementos $a_{1}, \cdots, a_{k}, b_{1}, \cdots b_{m}$, que conmutan mutuamente, la cerradura del ideal izquierdo en $E$ generado por $a_{1}, \cdots a_{k}$ es propio. Entonces existe $\lambda \in \mathbb{C}^{m}$ tal que el ideal izquierdo en $E$ generado por $a_{1}, \cdots, a_{k}, b_{1}-\lambda_{1}, \cdots b_{m}-\lambda_{m}$ también es propio.

Demostración. Demostremoslo para $m=1$. Sea $N=\overline{I_{E}^{l}\left(a_{1}, \cdots, a_{k}\right)}$ la cerradura del ideal izquierdo en $E$ generado por $a_{1}, \cdots, a_{k}$. Sea $b=b_{1} \in E$ que conmuta con todos los $a_{i}, 1 \leq i \leq k$, y $b \notin N$. Entonces existe una semi-norma submultiplicativa $\rho_{\alpha}$ tal que

$$
\left\{x \in E: \rho_{\alpha}(x-b)<\varepsilon\right\} \cap N=\emptyset \text { y }\left\{x \in E: \rho_{\alpha}(x-e)<\varepsilon\right\} \cap N=\emptyset,
$$

para algún $\varepsilon>0$. Consideremos

$$
\operatorname{ker} \rho_{\alpha}=\left\{x \in E: \rho_{\alpha}(x)=0\right\} .
$$


Por ser $\rho_{\alpha}$ semi-norma submultiplicativa en $E$, $\operatorname{ker} \rho_{\alpha}$ es un ideal bilateral cerrado de $E$. Así, el cociente $E / \operatorname{ker} \rho_{\alpha}$ es un álgebra normada, con norma $\|[a]\|=\rho_{\alpha}(a)$. Sea $E_{\alpha}$ la completación del álgebra normada $\left(E / \operatorname{ker} \rho_{\alpha},\|\cdot\|\right)$, entonces $E_{\alpha}$ es un álgebra de Banach compleja con unidad $[e]$.

Dado que $\operatorname{ker} \rho_{\alpha}$ es ideal bilateral cerrado en $E$ y $N$ es ideal izquierdo en $E$, entonces $N+\operatorname{ker} \rho_{\alpha}$ es ideal izquierdo en $E_{\alpha}$. Es claro que $\overline{N+\operatorname{ker} \rho_{\alpha}}$ es ideal en $E_{\alpha}$. Veamos que es propio. Supóngase que $E_{\alpha}=\overline{N+\operatorname{ker} \rho_{\alpha}}$, entonces $[e] \in \overline{N+\operatorname{ker} \rho_{\alpha}}$. Esto implica que existe una sucesión $\left\{\left[d_{l}\right]\right\} \subset\{[n]: n \in N\}=$ $N+\operatorname{ker} \rho_{\alpha}$ tal que

$$
\left\|\left[d_{l}\right]-[e]\right\| \rightarrow 0
$$

cuando $l \rightarrow \infty$. Así, dado $\delta<1$, existe $l_{0}$ número natural tal que

$$
\left\|\left[d_{l}\right]-[e]\right\|<\delta,
$$

si $l \geq l_{0}$. Por ser $E_{\alpha}$ álgebra de Banach con unidad, entonces $\left[d_{l}\right]$ es invertible en $E_{\alpha}$ con inverso $\left[d_{\alpha}^{-1}\right]$. En consecuencia $\left[d_{l}\right]\left[d_{l}^{-1}\right]=\left[d_{l} d_{l}^{-1}\right]=[e] \in N+\operatorname{ker} \rho_{\alpha}$. De donde existe $n \in N$ tal que $[n]=[e]$. Esto implica que $e-n \in \operatorname{ker} \rho_{\alpha}$. Por lo tanto ker $\rho_{\alpha}(e-n)=0$, lo cual es una contradicción.

De manera similar, al proceso previo, se puede probar que $[b] \notin \overline{N+\operatorname{ker} \rho_{\alpha}}$.

Ahora, como $a_{1}, \ldots, a_{k}, b \in E$ conmutan mutuamente, entonces sus correspondientes clases de equivalencia $\left[a_{1}\right], \ldots,\left[a_{k}\right],[b]$ también conmutan mutuamente. Consideremos el ideal izquierdo en $E_{\alpha}$ generado por los $\left[a_{i}\right], i \leq i \leq k$, y dado que $E_{\alpha}$ es un álgebra de Banach compleja con unidad, entonces, por el Teorema de Harte para álgebras de Banach complejas con unidad, existe $\lambda \in \mathbb{C}$ tal que

$$
(0, \ldots, 0, \lambda) \in S p_{E_{\alpha}}\left(\left[a_{1}\right], \ldots,\left[a_{k}\right],[b]\right) .
$$

Esto implica que el ideal izquierdo en $E_{\alpha}$ generado por $\left[a_{1}\right], \ldots,\left[a_{k}\right],[b]-$ $[\lambda e]$ es propio. Dado que el mapeo natural $E \rightarrow E / \operatorname{ker} \rho_{\alpha}$ es un homeomorfismo algebraico, entonces

$$
I_{E}^{l}\left(a_{1}, \ldots, a_{k}, b-\lambda e\right)
$$

es propio y el caso $m=1$ se sigue. La prueba se sigue aplicando inducción sobre $j, 1 \leq j \leq m$.

En [Mi; pags. 17-18] se probó el resultado siguiente

Proposición 5.1.2. Sea E un álgebra localmente convexa que satisface la siguiente propiedad: todo subconjunto de $E$ el cual es $m$-convexo, simétrico y cerrado, y cuyos múltiplos escalares cubren $E$, es una vecindad del cero de $E$. Además, supongamos que, para alguna base $\left\{U_{i}\right\}, i \in \Gamma$, para $E$, todo $x \in E$ tiene la propiedad : $x U_{i} \subset \lambda_{x_{i}} U_{i}$ ( $\lambda_{x_{i}}$ es un escalar que depende de $x$ e $i$ ), para todo $i$. Entonces $E$ es un álgebra localmente $m$ - convexa.

Como consecuencia, se tiene el siguiente

Corolario 5.1.3. Sea E un álgebra localmente convexa compleja con unidad que satisface las condiciones de la proposición anterior. Supongamos que para 
toda $(k+m)-$ upla de elementos $a_{1}, \ldots a_{k}, b_{1}, \ldots, b_{m} \in E$, que conmutan mutuamente, la cerradura del ideal izquierdo en $E$, generado por $a_{1}, \ldots a_{k}$, es propio. Entonces existe $\lambda \in \mathbb{C}^{m}$ tal que el ideal izquierdo en E, generado por $a_{1}, \ldots a_{k}, b_{1}-\lambda_{1}, \ldots, b_{m}-\lambda_{m}$, también es propio en $E$.

Usando Teorema 2.1.1, tenemos la fórmula del mapeo espectral para álgebras localmente $m-$ convexas.

Teorema 5.1.4. Sea E un álgebra localmente $m$ - convexa compleja con unidad. Sea $U$ una familia de ideales izquierdos en E cuya cerradura es propia. Entonces la familia $U$ tiene la propiedad de la proyección y el correspondiente espectro combinado

$$
S p_{U}(a)=\left\{\lambda \in \mathbb{C}^{k}: J \in U, a_{i}-\lambda_{i} e \in J, 1 \leq i \leq k\right\}
$$

donde $a=\left(a_{1}, \ldots, a_{k}\right)$ es una $k$ - upla de elementos de $E$ que conmutan mutuamente, satisface la fórmula del mapeo espectral ; es decir, para toda $k$ - upla de elementos que conmutan mutuamente y para todo mapeo polinomial $P: \mathbb{C}^{k} \rightarrow \mathbb{C}^{m}$ se tiene que

$$
P\left(S p_{U}(a)\right)=S p_{U}(P(a)) .
$$




\section{Bibliografía}

[AN] M. Akkar, C. Nacir, Continuité automatique dans les limites inductives localement convexes de Q-algèbres de Fréchet, Ann. Sci. Math. Quebec, 19 (1995).

[Ar] R. Arens, The algebra $L^{\omega}$ and convex topological rings, Bull. Amer.Math. Soc., 52 (1946), 931-935

[Bo] B. Bollobás, Linear Analysis, Cambridge Mathematical Text Books, 1999

[BD] F.F. Bonsall, J. Duncan, Complete Normed algebras, Springer Verlag Berlin, Heidelberg, NewYork, 1973

[Bou] N. Bourbaki, Espaces vectoriels topologiques, Chap. 1-2, Hermann, Paris, 1966

[Fr] M. Fragoulopoulou, Q-algebras: How close are they to Banach algebras? A survey, Proceedings of the international workshop held in Tartu, 1999, 125-134

[Ga] T. W. Gamelin. Uniform Algebras, Prentice-Hall, Englewood Cliffs, N.J., 1969

[GT] A. García-Máynez, A. Tamariz, Topología General, Editorial Porrúa 1988

[H1] R. Harte, Spectral mapping theorem, Proc. R.I.A., A 72 (1972) 89-107

[H2] R. Harte, The spectral mapping theorem in several variables, Bull. Amer. Math. Soc., 78 (1972), 871-875

[Hu] T. Husain, Multiplicative functionals on topological algebras, Pitman Advanced Publishing Program, 1983

[Ka] I. Kaplansky, Topological Rings, Amer. J. Math., 50 (1947), 153-183

[Kö] G. Köthe, Topological Vector Spaces I, Springer-Verlag,Berlin, 
1969

[Ma] A. Mallios, Topological Algebras. Selected Topics, North-Holland, 1986

[Mi] E. Michael, Locally multiplicatively-convex topological algebras, Mem. Amer. Math. Soc., 11 (1952)

[M1] V. Müller, Non-removable ideals in commutative Banach algebras, Studia Math., 74 (1982), 97-104

[M2] V. Müller, Spectral Theory of Linear Operators, Birkhäuser Verlag, Basel-Boston-Berlin, 2000

[Mo] D. Montgomery, Continuity in topological groups, Bull. Amer. Math. Soc., 42 (1936), 879-882

[Na] M. A. Naimark, Normed Algebras, Wolters-Noordhoff Publ., Groningen, 1972

[Pa] T. W. Palmer, Banach Algebras and the General Theory of *-algebras, Volume I: Algebras and Banach Algebras, Enciclopedy of Mathematics and its applications, Vol. 49, Cambridge University Press, 1994

[Ri] C. E. Rickart, General Theory of Banach Algebras, The University Series in Higher Mathematics,D. Van Nostrand Co., Princeton, N.J. Toronto-London-New York, 1960

[Ro] S. Rolewicz, Metric Linear Spaces, Warszawa, 1984

[Ru] W. Rudin, Functional Analysis, New York, 1973

[Sc] H. H. Schaefer, Topological Vector Spaces, Springer-Verlag, 1986

[Si] G. Silov, On the extension of maximal ideals, C. R. Acad. Sci. URSS, 24 No. 2 (1940), 83-84

[Sim] G. F. Simmons, Introduction to topology and modern analysis, Mc Graw Hill, 1965

[St] Z. Słodkowski, On ideals consisting of joint topological divisors of zero, Studia Math., 48 (1973), 83-88

[SŻ] Z. Słodkowski, W. Żelazko, On joint spectra of commuting families of operators, Studia Mathe., 50 (1974), 127-140

[So] A. Soltysiak, Joint spectra and Multiplicative Linear Functionals in Non-commutative Banach algebras, Adam Mickiewicz University Press, 1988

[Tk] V. Tkachuk, Curso básico de topología general, Universidad Autónoma Metropolitana, 1999

[Tr] F. Treves, Topological Vector Spaces, Distributions and Kernels, 
Academic Press, New York, 1967

[VW1] A. Velázquez González, A. Wawrzyńczyk, The projection property of a class of ideals in subalgebras of Banach algebras, Bol. Soc. Mat. Mex., 8 (2002), 24-29

[VW2] A. Velázquez González, A. Wawrzyńczyk, Spectral mapping formula for Waelbroeck algebras and their subalgebras, Contemporary Mathematics, Vol. 341 (2004),127-131

[Wa] L. Waelbroeck, Topological Vector Spaces and Algebras, Lec. Not. Math., 230, Springer-Verlag, 1971

[W1] A. Wawrzyńczyk, On ideals consisting of topological zero divisors Studia Math., 142 (3), 2000, 245-251

[W2] A. Wawrzyńczyk, Ideals of functions wich achieve zero on a compact set, Bol. Soc. Mat. Mex., 7 (3), 2001, 117-121

[W3] A. Wawrzyńczyk, The spectral mapping formula for Waelbroeck algebras, Submitted

[W4] A. Wawrzyńczyk, On subespectra generated by superalgebras, Bull. London Math. Soc., Submitted

[Ż1] W. Żelazko, On generalized topological divisors of zero in $m$-convex locally convex algebras, Studia Math., 28 (1966), 9-16

[Ż2] W. Żelazko, A characterization of Shilov boundary in function algebras, Comment. Math., 14 (1970), 59-64

[Ż3] W. Żelazko, Selected topics in Topological Algebras, Aarhus University Lecture Notes, 31, 1971

[Ż4] W. Żelazko, On a certain class of non-removable ideals in Banach algebras, Studia Math., 44 (1972), 87-92

[Ż5] W. Żelazko, Banach algebras, Amsterdam, 1973

[Ż6] W. Żelazko, On maximal ideals in commutative m-convex algebras, Studia Math., 58 (1976), 293-298

[Ż7] W. Żelazko, An axiomatic approach to joint spectra I, Studia Math., 64 (1979), 249-261

[Ż8] W. Żelazko, On ideal theory in Banach and topological algebras, Monografías del IMATE-UNAM, 1984

[Ż9] W. Żelazko, Some problems concerning $Q$-algebras, to appear 